\section{Kidney \\ Blood Pressure Research}

\title{
Mortality in Elderly Waiting-List Patients Versus Age-Matched Kidney Transplant Recipients: Where is the Risk?
}

\author{
Domingo Hernández ${ }^{\mathrm{a}} \quad$ Juana Alonso-Titos ${ }^{\mathrm{a}} \quad$ Ana María Armas-Padrón ${ }^{\mathrm{b}}$ \\ Pedro Ruiz-Esteban ${ }^{a} \quad$ Mercedes Cabello ${ }^{a}$ Verónica López ${ }^{a} \quad$ Laura Fuentes $^{a}$ \\ Cristina Jironda ${ }^{a}$ Silvia Ros ${ }^{a} \quad$ Tamara Jiménez $^{a} \quad$ Elena Gutiérrez $^{\mathrm{a}} \quad$ Eugenia Sola $^{\mathrm{a}}$ \\ Miguel Angel Frutos ${ }^{a} \quad$ Miguel González-Molina ${ }^{a} \quad$ Armando Torres $^{c}$ \\ aNephrology Department, Carlos Haya Regional University Hospital and University of Malaga, IBIMA, \\ Málaga, 'ba Cuesta Primary Health Care Centre, La Laguna, Tenerife, 'Nephrology Department, Hospital \\ Universitario de Canarias, CIBICAN, University of La Laguna, Tenerife and Instituto Reina Sofía de \\ Investigación Renal, IRSIN, Spain
}

\section{Key Words}

Elderly • Mortality • Waiting list • Kidney transplantation • Patient survival on dialysis • Risk factors

\begin{abstract}
The number of elderly patients on the waiting list (WL) for kidney transplantation (KT) has risen significantly in recent years. Because KT offers a better survival than dialysis therapy, even in the elderly, candidates for KT should be selected carefully, particularly in older waitlisted patients. Identification of risk factors for death in WL patients and prediction of both perioperative risk and long-term post-transplant mortality are crucial for the proper allocation of organs and the clinical management of these patients in order to decrease mortality, both while on the WL and after KT. In this review, we examine the clinical results in studies concerning: a) risk factors for mortality in WL patients and KT recipients; 2 ) the benefits and risks of performing KT in the elderly, comparing survival between patients on the WL and KT recipients; and 3) clinical tools that should be used to assess the perioperative risk of mortality and predict long-term post-transplant survival. The acknowledgment of these concerns could contribute to better management of high-risk patients and prophylactic interventions to prolong survival in this particular population, provided a higher mortality is assumed.
\end{abstract}




\section{Kidney Blood Pressure Research}

Hernández et al:: Mortality in Elderly Waiting-List and Kidney Transplant Patients

\section{Introduction}

Because nearly half of all incident end-stage renal disease (ESRD) patients worldwide are older than 65 years [1,2], nephrologists will need to decide which of these many elderly patients are suitable candidates for kidney transplantation (KT). In the current context of donor shortage, elderly patients (e.g. $>65$ years) are less frequently placed on the waiting list (WL), due to uremia-related life-threatening comorbidities, and, if they are listed, they have fewer chances of receiving a KT than their younger counterparts, partly owing to emergent events (cardiovascular disorders, infections, neoplasias, etc.) that can modify their transplant status whilst on the WL $[3,4]$. In addition, assessment of elderly KT candidates is complex and evidence-based clinical guidelines for the management of this particular population are lacking [4-8]. Moreover, many transplant teams are still reluctant to include elderly patients on the WL for KT as objective selection criteria for transplantation in this population are poorly defined $[3,4,9]$. Misconceptions regarding the inclusion criteria for elderly patients for KT could delay early referral to a transplant center or delay WL inclusion, leading to a longer time spent on dialysis.

Although many studies have demonstrated that KT is relatively safe and provides a better survival compared with age-matched dialysis patients [10-14], the benefits in survival could be tapered by risk in elderly patients, especially in those who have spent a prolonged time on the WL [3, 15-17]. Indeed, elderly KT recipients also have an increased risk of cardiovascular (CV) diseases, infections and neoplastic events, leading to higher post-transplant mortality than younger patients.

In this review, we focus on the clinical results of relevant reports concerning the benefits and risks of performing KT in elderly ESRD patients as opposed to remaining on the WL. We aim to answer the following questions: 1) Do we know the specific risk factors for mortality in elderly KT candidates?; 2) Is mortality in elderly patients on the WL for KT higher than in age-matched KT recipients?; and 3) What clinical tools should be used to assess the perioperative risk of mortality or to predict post-transplant survival in the long-term.

\section{Risks of death on the waiting-list}

Given the emerging evidence of the benefits of KT on survival in the elderly ESRD population, the age of patients on the WL for KT has risen significantly over the last decades worldwide $[3,4,18-21]$. However, previous reports have documented that approximately half of elderly KT candidates ( $>60$ years) in the United States die on the WL before receiving a deceased-donor transplant [22]. The annual mortality rate for all patients on the WL varies between $5-10 \%$ worldwide, but increases greatly in the older dialysis population [22, 23]. As expected, the most common causes of mortality in these patients during the first year on dialysis are CV disorders followed by infectious complications [21]. This higher mortality in elderly patients on the WL can be explained by the increasing comorbidities in this particular population. There are few clinical conditions that join together as many risk factors as uremia, especially in elderly patients. Indeed, the risk of death in older KT candidates increases significantly as their time on dialysis therapy increases. In the US population, several socio-demographic factors, such as older age, race, unemployment status, smoking, diabetes, CV comorbidity and prior history of admissions, are independent risk factors for mortality on dialysis therapy, including patients on the WL [24, 25]. Likewise, these same factors plus psychiatric disorders and a prior history of neoplasia have also been associated with an increased risk of death in European dialysis patients [26]. Unfortunately, not all are modifiable risk factors that can be addressed by targeted therapeutic interventions. Recently, competing risk models showed that older age, the presence of a central venous cathether at the start of hemodialysis therapy, unemployment and a Charlson Comorbidity index (CCI) score [27] higher than 3 were risk factors associated with mortality in Southern European 


\section{Kidney Blood Pressure Research}

KT candidates [21]. In addition, the presence of peripheral vascular disease (PVD) in patients on the WL has been related to a higher risk for mortality in large cohort studies of both the American and the European dialysis population [28-30]. In particular, PVD was associated with a 1.9-2.9 fold increased risk of mortality in wait-listed patients for KT. Furthermore, in KT candidates there may be a high prevalence of poor health indicators, recently established by American health authorities, such as obesity, physical inactivity, smoking, low income or low cultural levels, which could all increase the risk of death on the WL for KT [31, 32]. To further complicate this alarming situation, comorbidities can progress throughout ESRD [33], including those waitlisted for KT. Finally, other barriers to accessing KT, such as sociodemographic factors (obesity, female patients, patients who live in rural areas or far from transplant centers, among others) could increase the time on the WL and favor the onset or impairment of non-desirable comorbidities while still on the WL, leading to a higher mortality $[19,34-36]$. However, there may be a high overlap in the risk profile and life-expectancy of patients waitlisted for KT and those who are not [24], suggesting that accurate assessment of the risk of death should be performed in order to prioritize or properly assign a KT. Thus, early and accurate identification of risk factors for mortality, mainly modifiable risk factors, plus knowledge of WL inclusion criteria in elderly patients could help to target prophylactic interventions in this particular population. Consequently, comorbidity-based prediction of mortality in the short- and long-term could be crucial for making the best clinical decisions in elderly patients on the WL for KT, always considering that lack of KT is likely the biggest risk for dying in this particular population.

\section{Risk of mortality in elderly $\mathrm{KT}$ recipients}

A priori, elderly KT recipients have a greater risk of infections, CV events and neoplasia than younger patients. This is an obvious issue because ageing is associated with a higher risk of mortality in all populations. As expected, most reports have shown that the mortality risk increases with age, including in recipients from expanded criteria living kidney donors [37-41]. With ageing, an interaction between great CV comorbidity and infections and neoplastic processes within the framework of immunosuppressive therapy seems likely, leading to excessive post-transplant mortality [8, 42-44]. In addition, the risk of death in older KT recipients increases with increased comorbidity during the first post-transplant year $[39,45]$. Finally, older KT recipients are susceptible to cognitive impairment, poor functional status and frailty, increasing hospital readmission and mortality risk [46-50]. However, death-censored graft survival in the elderly, including KT recipients 80 years and older, appears to be comparable to or even better than that in younger KT recipients in the long term $[40,51,52]$. It is plausible, therefore, that, given the immunosenescence of the elderly, the use of lower doses of immunosuppressants and a more careful surveillance could minimize these life-threatening side effects, while still being able to provide adequate levels of immunosuppression. Given this background, careful selection of elderly candidates for KT based on a thoroughly comprehensive medical, psychosocial and functional evaluation would be recommendable, with emphasis on a CV work-up, to make the best clinical decisions in order to decrease post-transplant mortality, as reported [4]. Unfortunately, the optimal method of screening for $\mathrm{CV}$ diseases in these patients is still unclear.

\section{Comparing mortality risk on waiting-list versus post-transplantation}

An appropriate candidate for KT is a patient whose survival and quality of life (QOL) are expected to improve after KT as compared with remaining on dialysis. However, whether this holds true for all elderly WL patients is uncertain. For instance, consider a theoretical 83-year-old patient with advanced chronic renal disease secondary to nephrosclerosis, a non- 


\section{Kidney Blood Pressure Research}

Kidney Blood Press Res 2018;43:256-275

\begin{tabular}{l|l}
\hline DOI: $10.1159 / 000487684$ & (c) 2018 The Author(s). Published by S. Karger AG, Basel
\end{tabular}

Published onlıne: February 27, 2018

www.karger.com/kbr

smoker with no diabetes, hypertension, or prior cardiovascular disease, with a functioning arterio-venous fistula, hemoglobin blood levels of $12 \mathrm{~g} / \mathrm{dL}$ and CCI of 6 points (Patient A); would it be safe for this patient to receive a KT? Would the likelihood of death during dialysis be greater than after a KT?. For practical purposes and in another clinical setting, let us also consider a hypothetical 80-year-old patient with renal disease secondary to insulindependent diabetes, former smoker, and hypertension, a healed myocardial infarction of 6 months previously, a central venous catheter for hemodialysis and CCI of 12 points (Patient B). What would be the likelihood of death during dialysis and after a KT for this patient?

Based on a recent review on criteria for and appropriateness of KT in elderly patients, the European Renal Association-European Dialysis and Transplant Association (Descartes Working Group and European Renal Best Practice) recommends KT for carefully selected ESRD patients because KT is superior to dialysis in terms of patient survival, QOL and cost-effectiveness, as evidenced by multiple studies carried out in older ESRD patients [7, 11-13, 16, 52-59], including those on home-dialysis therapy [60]. This potential benefit of KT can vary greatly between countries. Indeed, survival on dialysis appears to be better in Europe than the US. Thus, the expected survival benefit with transplantation could be lower in Europe compared with the US [61,62]. Additionally, virtually all guidelines agree that patients should not be deemed ineligible for KT based on age alone [4]. However, prolonged waiting times dramatically decrease the clinical and economic benefits of KT, suggesting that living donor transplantation may be of particular benefit in this population [16].

Focusing on patients waitlisted for KT, observational studies have shown that the mortality risk is significantly higher in the WL patients than in age-matched KT recipients, regardless of the type of transplant, although these differences were mainly observed beyond the third month after KT $[9,11-14,56,63]$. Additionally, two recent observational studies have shown that utilization of kidneys with a high Kidney Donor Profile Index (>85\%) or deceased diabetic donor kidneys also provides a higher survival in older recipients compared with waitlisted patients $[64,65]$. However, an elegant Dutch observational study performed in elderly KT patients receiving deceased-donor grafts from elderly donors $(\geq 65$ years) demonstrated an increased mortality risk compared with waitlisted elderly patients remaining on dialysis [66]. A higher incidence rate of delayed graft function, impaired renal function and rejection in elderly recipients who receive kidneys from elderly donors could have explained this concerning finding. Table 1 shows the survival benefit for KT recipients over potential candidate patients for KT in several observational studies, including the elderly population.

A few years ago, Tonelli et al. performed a systematic review of cohort studies involving 110 eligible studies with a total of 1,922, 300 participants and comparing outcomes between chronic dialysis patients and KT recipients [67]. Most studies found a significantly lower mortality as well as a lower rate of CV events associated with KT. Additionally, the magnitude of the clinical benefits seemed to increase over time. More interestingly, similar findings were observed in the subset of 10 studies including 97, 873 waitlisted patients, supporting the clinical benefit of KT compared with dialysis therapy, regardless of age and comorbidity. Similarly, a recent cohort study of the Danish Nephrology Registry showed a significantly better survival in KT recipients compared with waitlisted patients despite high comorbidity [17]. Nonetheless, it is possible that the beneficial effect on survival of KT is not so robust in patients aged $>70$ years, diabetics and those with chronic obstructive pulmonary disease, as previously reported by Perez-Saez et al. [68], suggesting that this benefit may not be extrapolatable to older patients. However, a later systematic review carried out by these same authors showed an overall better patient survival at the fifth year post-transplantation in elderly KT recipients compared with age-matched waitlisted patients [69].

In summary, mortality risk is significantly higher in WL patients than in age-matched KT recipients, especially in elderly patients. Transplanting with low-quality allografts could lead to higher mortality, which underlines the importance of proper selection and preservation if the allocation of elderly donors to elderly recipients is to be expanded. 


\section{Kidney Blood Pressure Research}

Table 1. Observational studies comparing the benefit on survival of a kidney transplant over potential candidate patients for transplantation, including elderly population. Abbreviations: Tx, transplantation; WL, waiting-list; SCD, standard cadaveric donor; ECD, expanded cadaveric donor; LD, living donor; DDT, deceased donor transplant; KDPI, Kidney Donor Profile Index

\begin{tabular}{|c|c|c|c|c|}
\hline Reference & $\begin{array}{c}\text { Transplant } \\
\text { period }\end{array}$ & Recipient population & $\begin{array}{c}\text { Transplanted vs. } \\
\text { Waitlisted patients }\end{array}$ & Patient survival or mortality risk \\
\hline $\begin{array}{l}\text { Wolfe et al.,[11] } \\
1999\end{array}$ & $1991-1997$ & All ages & $23275 / 22889$ & $\begin{array}{l}\text { Adjusted mortality risk Tx vs. WL: age } 40-59: 0.3 \\
\text { (95\% CI: 0.3-0.4); Age 60-74 y: } 0.4 \text { (95\% CI 0.3-0.5) }\end{array}$ \\
\hline $\begin{array}{l}\text { Ojo et al., [12] } \\
2001\end{array}$ & $1992-1997$ & All ages & $41892 / 71295$ & $\begin{array}{c}\text { Adjusted } 5 \text { y-survival: ideal Tx } 85 \% \text {; marginal Tx } \\
\text { 75\%: Adjusted mortality Tx vs WL: ideal Tx 0.5; } \\
\text { marginal Tx } 0.7(P=0.001)\end{array}$ \\
\hline $\begin{array}{l}\text { Rao et al., }[13] \\
2007\end{array}$ & $1990-2004$ & $>70 \mathrm{y}$ & $2436 / 3229$ & $\begin{array}{l}\text { At } 4 \text { y-survival, TX: } 66 \% \text { (overall adjusted mortality } \\
\text { risk: } 0.6,95 \% \text { CI } 05-0.7 \text { ); WL: } 51 \%\end{array}$ \\
\hline $\begin{array}{l}\text { Heldal et al., [56] } \\
2010\end{array}$ & $1990-2005$ & $>70 \mathrm{y}$ & $233 / 53$ & $\begin{array}{c}\text { Period } 1990-99 \text { : Survival at } 1^{\text {st }}, 2^{\text {nd }} \text { and } 5^{\text {th }} \text { year: Tx } \\
79 \%, 60 \% \text { and } 39 \% \text { vs. WL } 93 \%, 66 \%, 29 \% \\
\text { Period } 2000-2005: \text { Tx } 89 \%, 74 \%, 64 \% \text { vs. WL } 98 \% \text {, } \\
56 \%, 33 \%\end{array}$ \\
\hline $\begin{array}{l}\text { Gill et al., [71] } \\
2013\end{array}$ & $1995-2007$ & $\begin{array}{l}\geq 65 \mathrm{y} \text {. } \\
\text { Low, intermediate } \\
\text { and high CV risk }\end{array}$ & $11072 / 4396$ & $\begin{array}{c}\text { Mortality risk ECD >SCD >LD Days to equal survival } \\
\text { Tx/WL: High risk patients: LD } 130 \text { days, SCD } 368 \\
\text { days; ECD } 521 \text { days. }\end{array}$ \\
\hline $\begin{array}{l}\text { Sorensen et al, } \\
\text { [17] } 2016\end{array}$ & $1995-2011$ & All ages & $2349 / 825$ & $\begin{array}{l}\text { Adjusted mortality risk for elderly (>65 y) Tx } \\
\text { patients receiving DDT: } 0.45 \text { ( } 95 \% \text { CI } 0.26-0.75 \text { ) }\end{array}$ \\
\hline $\begin{array}{l}\text { Pérez-Sáez et al, } \\
\text { [68] } 2016\end{array}$ & $1990-2013$ & All ages & $389 / 2040$ & $\begin{array}{l}\text { Overall mortality risk for Tx: } 0.44 \text { (95\% CI } 0.32 \text { - } \\
0.62) \text {; if recipient 65-69 y: HR } 0.56 \text { (95\% CI 0.34- } \\
0.92)\end{array}$ \\
\hline $\begin{array}{l}\text { Peters-Sengers } \\
\text { et al., [66] } 2017\end{array}$ & $2002-2012$ & $\begin{array}{l}<65 \text { y }(\mathrm{N}=2883) \text { and } \\
\quad \geq 65 \text { y }(\mathrm{N}=714)\end{array}$ & $3597 / 504$ & $\begin{array}{c}5 \text {-year mortality elderly patients (>65y): Tx } 60 \% \\
\text { vs WL } 61.3 \%\end{array}$ \\
\hline $\begin{array}{l}\text { Chen et al., }{ }^{[63]} \\
2017\end{array}$ & $2006-2013$ & $>70 \mathrm{y}$ & $2397 / 79681$ & $\begin{array}{l}\text { 5-year mortality: low risk patients (scoring 1): Tx } \\
19 \% \text { vs WL 50\%; high risk patients (scoring 5): Tx } \\
40 \% \text { vs WL } 90 \%\end{array}$ \\
\hline $\begin{array}{l}\text { Jay et al., [64] } \\
2017\end{array}$ & $2003-2012$ & $>60 y$ & $4359 / 74394$ & $\begin{array}{l}\text { Adjusted } 1-2 \text { year mortality risk using donor with } \\
\text { KDPI score }>85 \% \text { : } 0.52 \text { (95\% CI } 0.45-0.61)\end{array}$ \\
\hline $\begin{array}{l}\text { Cohen et al., [65] } \\
2017\end{array}$ & $>1994$ & All ages (>18 y.) & $134,661 / 302,958$ & $\begin{array}{l}\text { Adjusted mortality risk in overall cohort with } \\
\text { diabetic donor: Tx } 0.91 \text { (95\% CI 0.84-0.98) }\end{array}$ \\
\hline
\end{tabular}

\section{Assessment of perioperative risk in elderly candidates for KT}

Cardiovascular diseases are the most common cause of death in KT recipients, with the highest rates in the peritransplant period. Older age is considered a very important risk factor for mortality in the perioperative and early post-transplant period in comparison with age-matched waitlisted dialysis patients, and this risk can increase greatly according to the quality of graft $[66,70,71]$. Thus, potential elderly candidates for KT should have a careful medical evaluation of their CV comorbidities. Furthermore, evaluation of risk in the peritransplant period can be crucial to properly assign a KT. Accordingly, an important issue for older patients undergoing a transplant assessment is their likelihood of an unfavorable perioperative outcome. Most guidelines recommend that patients with either a reduced life-expectancy or high perioperative risk of death should not be waitlisted for KT, but this principle is often not easy to apply in daily clinical practice [72]. Indeed, multiple prognostic indices are available to assess perioperative mortality risk. These have been validated in both the general population and renal patients, but no clear guidelines on the criteria for their use in patients waitlisted for KT are currently available. Moreover, there is limited information on the use of these scores for predicting the risk of early death in elderly candidates for KT. Accurate assessment of the perioperative surgical risk could allow us to undertake a careful selection of waitlisted patients in order to minimize early mortality post-transplantation. But do we have clinical tools to accurately estimate early mortality 


\section{Kidney \\ Blood Pressure Research}

Table 2. Surgical risk scores frequently used in the general population. *Dialysis patients without other comorbidities are considered as ASA III

\begin{tabular}{|c|c|}
\hline Risk score & Assessment/Risk stratification \\
\hline $\begin{array}{l}\text { The ASA classification* } \\
\text { The American Society of Anesthesiologists } \\
\text { Fitz-Henry, J Ann R Coll Surg Engl 2011; 93: 185-187 }\end{array}$ & $\begin{array}{c}\text { Assessment: ASA I - ASA VI } \\
\text { Scoring based on: } \\
\text { - } \quad \text { Normal healthy patients (ASA I) } \\
\quad \text { Systemic disease mild (ASA II) or severe (ASA III) } \\
\quad \text { Life-threatening systemic disease (ASA IV) } \\
\qquad \quad \text { Moribund patients (ASA V) } \\
\quad \text { Deceased-donor (ASA VI) }\end{array}$ \\
\hline $\begin{array}{l}\text { Revised Cardiac Risk Index } \\
\text { Lee TH, Circulation. 1999;100:1043-1049 } \\
\text { https://www.mdcalc.com/revised-cardiac-risk-index-pre-operative-risk }\end{array}$ & $\begin{array}{l}\text { Assessment: Class I (very low risk) - Class IV (high risk) } \\
\text { Scoring based on: } \\
\bullet \quad \text { High risk surgery } \\
\text { Cardiovascular disease: coronary artery disease, } \\
\text { congestive heart failure, cerebrovascular disease } \\
\bullet \quad \text { Diabetes on insulin } \\
\bullet \quad \text { Serum creatinine }>2 \mathrm{mg} / \mathrm{dL}\end{array}$ \\
\hline $\begin{array}{l}\text { American College of Surgeons (ACS) } \\
\text { Winoker JS, 2017; J Urol 2017 } \\
\text { https://riskcalculator.facs.org/RiskCalculator/ }\end{array}$ & $\begin{array}{c}\text { Assessment: Percent risk - chance of outcome - predicted } \\
\text { length of hospital stay } \\
\text { Scoring based on: } \\
\text { Surgical procedure } \\
\text { - Age, gender, ASA class, steroid use, comorbidities } \\
\text { (diabetes, hypertension, sepsis, cancer, CHF, COPD, } \\
\text { acute renal failure), dialysis, smoker, BMI }\end{array}$ \\
\hline $\begin{array}{l}\text { Gupta Index: perioperative myocardial infarction or cardiac arrest risk } \\
\text { calculator }\end{array}$ & $\begin{array}{l}\text { Assessment: percentile of risk/percent risk } \\
\text { Scoring based on: }\end{array}$ \\
\hline $\begin{array}{l}\text { Gupta PK, Circulation. 2011;124:381-387 } \\
\text { https://www.qxmd.com/calculate/calculator_245/gupta-perioperative- } \\
\text { cardiac-risk }\end{array}$ & $\begin{array}{l}\text { - Age, ASA class, serum creatinine, functional status, } \\
\text { surgical procedure }\end{array}$ \\
\hline $\begin{array}{l}\text { Detsky's Modified Cardiac Risk Index } \\
\text { Detsky AS 1986, J Gen Intern Med 1; } 213 \\
\text { http://www.fpnotebook.com/CV/Surgery/DtskysMdfdCrdcRskIndx.htm }\end{array}$ & $\begin{array}{c}\text { Assessment: Class } 1 \text { (low risk), class } 2 \text { (moderate risk), class } \\
3 \text { (high risk) } \\
\text { Scoring based on: } \\
\bullet \quad \text { Age } \\
\text { Cardiac conditions: prior MI, angina pectoris, } \\
\text { pulmonary edema, critical aortic stenosis } \\
\bullet \quad \text { General medical status }\end{array}$ \\
\hline $\begin{array}{l}\text { Surgical Outcome Risk Tool (SORT) } \\
\text { K.L. Protopapa KL, 2014; Br J Surg 101; 1774-1783. } \\
\text { The National Confidential Enquiry into Patient Outcome and Death (NCEPOD) } \\
\text { www.ncepod.org.uk }\end{array}$ & $\begin{array}{c}\text { Assessment: percent risk (high risk }>5 \% \text {; critical care if } \\
\geq 10 \% \text { ) } \\
\text { Scoring based on: } \\
\qquad \quad \text { Procedure, severity, ASA class, urgency } \\
\bullet \quad \text { Thoracic, gastrointestinal or vascular surgery } \\
\bullet \quad \text { Cancer } \\
- \text { Age }\end{array}$ \\
\hline
\end{tabular}

in this particular population? Table 2 shows some surgical risk scores frequently used in the general population, which could be applied in elderly waitlisted patients, showing the clinical information necessary for inclusion on the list and, thus, to calculate the risk [73-78]. Although these risk indices are not universally applied in daily clinical transplant practice, efforts should made to apply those indices involving renal impairment, especially when assessing death risk in elderly patients before performing KT. For instance, applying the American Society of Anesthesiology (ASA) classification to assess the perioperative risk based on comorbidity conditions and renal disease of our 83-year-old Patient A mentioned above would give an intermediate risk, but not a high risk [73]. By contrast, this same classification would give a higher perioperative risk for our Patient B (Table 3). The Revised Cardiac Index, which includes age, cardiac disorders and renal function, assigns our Patient A a total score of 2; that is, an intermediate and assumable perioperative risk (https://www.mdcalc.com/revised-cardiac-risk-index-pre-operative-risk) [74]. However, this risk score assigns our Patient B a total score of 3 (high perioperative risk). The Surgical Risk Calculator of the American College of Surgeons includes dialysis therapy as well as age and other comorbid conditions in patients who are due to undergo major surgery, as is transplant (https://riskcalculator.facs.org/RiskCalculator/) [75]. A priori, our elderly Patient A would have a moderate risk of early death post-transplantation, but not a high risk. However, our Patient B would have a moderate-high risk of early death post-transplantation (Table 3). Finally, the Surgical Outcome Risk Tool (SORT score) includes age, comorbidities and the ASA classification (www.ncepod.org.uk) [78]. Again, our Patient A would have an intermediate and manageable perioperative risk whereas our Patient B would have a high mortality risk. Thus, assessing perioperative risk at the time of listing may help determine the advisability of undergoing transplant or continuing on dialysis therapy. Accordingly, it is plausible to perform a KT in carefully selected elderly waiting-list patients, using risk indices 


\section{Kidney \\ Blood Pressure Research}

Table 3. Risk stratification in our two different typical risk patients according to several prediction models. WL, waiting-list; TX, transplantation; ASA, The American Society of Anesthesiology; CCI, Charlson comorbidity index

\begin{tabular}{|c|c|c|}
\hline Application of Risk score & Risk stratification Patient A & Risk stratification Patient B \\
\hline \multicolumn{3}{|l|}{ Perioperative risk } \\
\hline The ASA Classification & $\begin{array}{c}\text { ASA classification: } \\
\text { intermediate risk (ASA III) }\end{array}$ & $\begin{array}{l}\text { ASA classification: high risk } \\
\text { (ASA IV) }\end{array}$ \\
\hline \multicolumn{3}{|l|}{$\begin{array}{l}\text { The American Society of Anesthesiologists } \\
\text { Fitz-Henry, J Ann R Coll Surg Engl 2011;93:185-187 }\end{array}$} \\
\hline $\begin{array}{l}\text { Revised Cardiac Risk Index } \\
\text { Lee TH, Circulation. 1999;100:1043-1049 } \\
\text { https://www.mdcalc.com/revised-cardiac-risk-index-pre-operative-risk }\end{array}$ & $\begin{array}{l}\text { Points 2: Class III, } \\
\text { Intermediate perioperative } \\
\text { risk (6.6\% complications) }\end{array}$ & $\begin{array}{l}\text { Points 3: Class IV } \\
\text { High perioperative risk }(>11 \% \\
\text { complications) }\end{array}$ \\
\hline \multicolumn{3}{|l|}{ ACS NSQIP ${ }^{\circledR}$ Surgical Risk Calculator } \\
\hline \multicolumn{3}{|l|}{$\begin{array}{l}\text { https://riskcalculator.facs.org/RiskCalculator/ } \\
\text { Gupta Index: perioperative myocardial infarction or cardiac arrest } \\
\text { (MICA) risk calculator }\end{array}$} \\
\hline $\begin{array}{l}\text { Gupta PK, Circulation. 2011;124:381-387 } \\
\text { https://www.qxmd.com/calculate/calculator_245/gupta-perioperative- } \\
\text { cardiac-risk }\end{array}$ & $\begin{array}{l}\text { Estimated risk probability for } \\
\text { perioperative MICA } 0.56 \%\end{array}$ & $\begin{array}{l}\text { Estimated risk probability for } \\
\text { perioperative MICA } 1.4 \%\end{array}$ \\
\hline $\begin{array}{l}\text { Detsky's Modified Cardiac Risk Index } \\
\text { Detsky AS 1986, J Gen Intern_Med 1; } 213 \\
\text { http://www.fpnotebook.com/CV/Surgery/DtskysMdfdCrdcRskIndx.htm } \\
\text { Surgical Outcome Risk Tool (SORT) }\end{array}$ & Class 1: Points 0 -15 Low risk & $\begin{array}{l}\text { Class 2: Points } 20-30 \\
\text { Intermediate risk }\end{array}$ \\
\hline $\begin{array}{l}\text { K.L. Protopapa KL, 2014; Br J Surg 101; 1774-1783. } \\
\text { The National Confidential Enquiry into Patient Outcome and Death } \\
\text { (NCEPOD) www.ncepod.org.uk }\end{array}$ & Medium mortality risk (3.7\%) & High mortality risk ( $>5 \%)$ \\
\hline Risk Prediction in Dialysis or WL patients, and transplant patients & $\begin{array}{l}\text { Low comorbidity }(\mathrm{CCI}<5) \\
5 \text {-year post-TX expected }\end{array}$ & $\begin{array}{l}\text { High comorbidity }(\mathrm{CCI} \geq 5) \\
\text { 5-year post-TX expected }\end{array}$ \\
\hline Wu et al., ${ }^{[111]} 2005$ & survival $90 \%$ & survival $70 \%$ \\
\hline Hernandez et al., ${ }^{[108]} 2005$ & 5-y survival post-TX: $78 \%$ & $5-y$ survival post- TX: $70 \%$ \\
\hline van Walraven et al., [94] 2010 & $\begin{array}{c}\text { WL: } 5 \text {-y expected survival } \\
30 \% \\
\text { TX: } 5 \text {-y expected survival } \\
60 \%\end{array}$ & $\begin{array}{l}\text { WL: 5-y expected survival } 20 \% \\
\text { TX: } 5 \text {-y expected survival } 45 \%\end{array}$ \\
\hline Grams et al., [32] 2012 & 3-y survival post-TX: 75\% & 3 -y survival post-TX: $69 \%$ \\
\hline Floege et al., [98] 2015 & $\begin{array}{l}\text { Intermediate mortality risk } \\
\text { on dialysis: } 1 \text {-year mortality } \\
\text { 9\%; } \\
\text { 2-year mortality } 20 \%\end{array}$ & $\begin{array}{l}\text { High mortality risk on dialysis: } \\
\text { 1-year mortality } 20 \% \text {; 2-year } \\
\text { mortality } 35 \%\end{array}$ \\
\hline Dusseux et al., [100] 2015 & $\begin{array}{l}\text { WL: 3-y expected survival } \\
\text { 70\% (Score 6) } \\
\text { May be suitable for TX } \\
\text { evaluation }\end{array}$ & $\begin{array}{l}\text { WL: 3-y expected survival } 43 \% \\
\text { (score 10) } \\
\text { May not be suitable for TX } \\
\text { evaluation }\end{array}$ \\
\hline Couchoud et al., [99] 2015 & $\begin{array}{l}\text { Low risk (score 4) } \\
\text { 3-month expected mortality } \\
\text { after initiating dialysis 5\% }\end{array}$ & $\begin{array}{l}\text { Intermediate risk (score } 12 \text { ) } \\
\text { 3-month expected mortality } \\
\text { after initiating dialysis } 24 \%\end{array}$ \\
\hline Laging et al., [124] 2016 & 5 -year post-TX mortality $6 \%$ & 5-year post-TX mortality $30 \%$ \\
\hline Lorent et al., [125] 2016 & $\begin{array}{l}\text { The cumulative probability of } \\
\text { death after the } 1 \text { st } \mathrm{y} \text {. post-TX } \\
\text { is } 0.46\end{array}$ & $\begin{array}{c}\text { The cumulative probability of } \\
\text { death after the } 1^{\text {st }} y \text {. post-TX is } \\
0.26\end{array}$ \\
\hline Chen et al., [63] 2017 & $\begin{array}{l}\text { WL: } 5 \text {-year mortality } 60 \% \text {, } \\
\text { score }-4 \text { (suitable for } \\
\text { transplant) }\end{array}$ & $\begin{array}{l}\text { WL: } 5 \text {-year mortality } 80 \% \text {, } \\
\text { score } 4 \text { (a priori no suitable for } \\
\text { transplant) }\end{array}$ \\
\hline & $\begin{array}{l}\text { Transplant: 5-year mortality } \\
\text { 1-year post-TX mortality }\end{array}$ & Transplant: 5-year mortality \\
\hline Molnar et al., [127] 2017 & $\begin{array}{c}12 \% \\
5 \text {-year post-TX mortality } \\
28 \%\end{array}$ & $\begin{array}{l}\text { 1-year post-TX mortality } 16 \% \text {; } \\
\text { 5-year post-TX mortality } 50 \%\end{array}$ \\
\hline
\end{tabular}

that involve renal impairment, provided an increase in early post-operative risk is accepted. Nevertheless, prospective studies in elderly WL patients incorporating a more detailed risk prediction based on preoperative risk scores and graft quality scores are needed. 


\section{Kidney Blood Pressure Research}

Kidney Blood Press Res 2018;43:256-275

\begin{tabular}{l|l}
\hline DOI: $10.1159 / 000487684$ & (c) 2018 The Author(s). Published by S. Karger AG, Basel
\end{tabular}

Published online: February 27, 2018 www.karger.com/kbr

\section{Prediction of mortality and comorbidity in waiting-list patients and kidney transplant recipients}

Prediction of mortality and comorbidity, such as ischemic heart disease (IHD), in both waitlisted patients and KT recipients may be crucial for performing the most timely therapeutic measures in order to decrease mortality in these patients. Accordingly, prognostic indices should include comorbidity risk factors as well as secondary survival measures to estimate survival accurately and to make targeted therapeutic decisions or to guide eligibility for KT properly. The CCI has been considered a useful tool for predicting the risk of mortality in both the general population and renal patients, but it does not incorporate other uremia-related risk factors that can have a negative impact on survival [27, 79]. Similarly, the Framingham risk score, which is widely used in the general population, can also underestimate the CV risk in renal patients, especially in those with poor health indicators [80]. Consequently, several comorbidity scores have been proposed to predict mortality in renal patients $[63,79,81-$ 103] (Table 4). However, most previous studies have reported models that are difficult to use, based on odds ratios or hazard ratios from many variables. In addition, most of these lack a mortality analysis exclusively for elderly patients (e.g., >70 years) on the WL or mostly do not assess simultaneously mortality risk after KT as compared with remaining on the $\mathrm{WL}$. Thus, further studies are clearly needed to determine the accuracy of predictive tools based on common clinical parameters and to relate their performance to more sophisticated predictors of clinical outcome. With this framework, a large Canadian cohort study developed and validated a prediction model to assess simultaneously survival in three patient groups: patients on the WL, patients who could receive a deceased-donor KT, and potential KT recipients from a living donor. After sample randomization in two subpopulations, a score was obtained from a Cox multivariate analysis, giving a total risk score for each patient, such that as the risk score increases, the risk of death increases exponentially after 5 years [94]. Applying this risk score, the 5-year expected survival in our elderly Patient A would be $30 \%$ if he remains on the WL. By contrast, a survival of $60 \%$ would be expected if this patient receives a deceased-donor KT. The 5-year expected survival rates for our Patient B would be $20 \%$ and $45 \%$ if he remains on the WL or if he receives a successful deceased-donor KT, respectively (Table 3). More recently, Floege et al. developed a mortality risk score in 11, 508 European incident hemodialysis patients with a mean age of 64 years using covariates such as age, smoking, body mass index (BMI), vascular access, comorbidities and certain biochemical parameters. Patients were stratified into a low, intermediate or high risk accordingly, but not all patients were on the WL or were elderly [98], which could be an important limitation for applying this risk score in elderly waitlisted patients. Nonetheless, based on this risk score our Patient A would have an intermediate risk of death at the first and second posttransplant year ( $9 \%$ and $20 \%$, respectively). By contrast, our Patient B would have a high risk of death in the same post-transplant periods (20\% and $35 \%$, respectively). Couchoud et al. also developed a mortality risk score in a large cohort of elderly hemodialysis patients (>75 years old) from the National French Registry based on age, gender, frailty, serum albumin, BMI and other comorbidities, classifying patients into a low, intermediate or high risk of death [99]. The 3-month expected mortality after initiating dialysis in our Patient $\mathrm{A}$ is $5 \%$ (low risk), whereas our Patient B would have a higher mortality risk after starting dialysis therapy (24\%) (Table 3). Similarly, a large French cohort study carried out in 16, 337 elderly dialysis patients ( $>70$ years old) developed a risk score for death post-inclusion on the WL using age, gender, diabetic condition, mobility status, BMI and comorbidities [100]. The probability of patients being alive within 3 years after listing was around $70 \%$ for the lowest quintile (0-6 points), representing about $20 \%$ of incident patients, as in our Patient A. By contrast, the 3-year expected survival of our Patient B would only be $43 \%$ (score 10 ), and, thus, he would not be suitable for transplant evaluation (Table 3). Finally, Chen et al. recently created a 5-year mortality prediction score in elderly (70 years or older) incident dialysis patients, potentially suitable candidates for KT, from the United States Renal Data System. 


\section{Kidney Blood Pressure Research}

Table 4. Comorbidity indices to predict mortality in renal patients. Abbreviations: ARO, Analyzing Data, Recognizing Excellence and Optimizing Outcomes; AUC, area under curve; HD, hemodialysis; CCI, Charlson comorbidity index; CHADS2, Score for atrial fibrillation stroke risk; DOPPS, Dialysis Outcomes Practice Patterns; HR, Hazard ratio; ICED, index of coexistent disease; OR, odd ratio; KT, kidney transplantation; NHIRD, Nation Health Insurance system of Taiwan; PD, peritoneal dialysis; REMIS, Renal Management Information System; REIN, French National Renal Epidemiology and Information Network; UNOS, United Network for Organ Sharing transplant; USRDS, United States Renal Data System

\begin{tabular}{|c|c|c|c|c|}
\hline Reference/year & $\begin{array}{l}\text { Study data/ } \\
\text { Patients }\end{array}$ & Population & Variables & $\begin{array}{l}\text { Assessment/ } \\
\text { Risk } \\
\text { stratification }\end{array}$ \\
\hline $\begin{array}{l}\text { Hutchinson et al.,[81] } \\
1982\end{array}$ & $\begin{array}{l}\text { Multicenter } \\
\quad \mathrm{N}=220\end{array}$ & Starting dialysis & $\begin{array}{l}\text { Age, length of diabetes, left } \\
\text { ventricular failure }\end{array}$ & $\begin{array}{c}\text { Low }(<30) \\
\text { medium }(30- \\
70) \text {, high }(>70)\end{array}$ \\
\hline $\begin{array}{l}\text { Wright [82] } \\
1991\end{array}$ & $\begin{array}{l}\text { Single center } \\
\quad \mathrm{N}=138\end{array}$ & HD & Age and comorbidity & $\begin{array}{l}\text { Low-medium- } \\
\text { high }\end{array}$ \\
\hline $\begin{array}{l}\text { Khan et al., [83] } \\
1993\end{array}$ & $\begin{array}{l}\text { Single center } \\
\quad \mathrm{N}=375\end{array}$ & HD & Age, diabetes and comorbidity & $\begin{array}{l}\text { Low-medium- } \\
\text { high }\end{array}$ \\
\hline $\begin{array}{l}\text { Davies et al.,[84] } \\
1995\end{array}$ & $\begin{array}{l}\text { Single center } \\
\mathrm{N}=97\end{array}$ & PD & Age, comorbidity, albumin, & $\begin{array}{l}\text { Low-medium- } \\
\text { high }\end{array}$ \\
\hline $\begin{array}{l}\text { Barrett et al.,[85] } \\
1997\end{array}$ & $\begin{array}{c}\text { Multicenter } \\
\mathrm{N}=822\end{array}$ & Starting dialysis & Age, comorbidity & $\begin{array}{l}\text { Low }(0-4) \\
\text { medium }(5-9), \\
\text { high }(>9)\end{array}$ \\
\hline $\begin{array}{l}\text { Fried et al.,[86] } \\
2001\end{array}$ & $\begin{array}{l}\text { Single center } \\
\quad \mathrm{N}=268\end{array}$ & PD & Age, comorbidity, albumin & $\begin{array}{l}\mathrm{HR} \text {, increase in } \\
\text { the CCI }\end{array}$ \\
\hline $\begin{array}{l}\text { Beddhu et al., }{ }^{[87]} \\
2002\end{array}$ & $\begin{array}{l}\text { Single center } \\
\quad \mathrm{N}=97\end{array}$ & PD & Age. comorbidity, & $\begin{array}{l}\mathrm{HR} \text {, increase in } \\
\text { the CCI }\end{array}$ \\
\hline $\begin{array}{l}\text { Van Manen et al.,[88] } \\
2002\end{array}$ & $\begin{array}{l}\text { Multicenter } \\
\mathrm{N}=1205\end{array}$ & Starting dialysis & Comorbidity & $\begin{array}{l}\text { Low-medium- } \\
\text { high }\end{array}$ \\
\hline $\begin{array}{l}\text { Van Manen et al.,[79] } \\
2003\end{array}$ & $\begin{array}{c}\text { Multicenter } \\
\mathrm{N}=1041\end{array}$ & Starting dialysis & $\begin{array}{l}\text { Comorbidity (Khan, Davies and } \\
\text { CCI indices) }\end{array}$ & $\begin{array}{l}\text { Low-medium- } \\
\text { high }\end{array}$ \\
\hline Miskulin et al.,[89] 2003 & $\begin{array}{l}\text { Multicenter } \\
\mathrm{N}=1772\end{array}$ & HD & 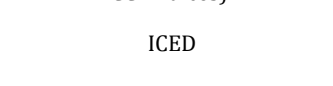 & $\begin{array}{l}\text { Low (0-1), } \\
\text { Medium (2), } \\
\text { High (3) }\end{array}$ \\
\hline $\begin{array}{l}\text { Miskulin et al.,[90] } \\
2004\end{array}$ & $\begin{array}{l}\text { Multicenter } \\
\mathrm{N}=1779\end{array}$ & HD & ICED, Wright-Khan, Davies, CCI, & AUC \\
\hline $\begin{array}{l}\text { Hemmelgarn et al.,[91] } \\
2003\end{array}$ & $\begin{array}{l}\text { Single center } \\
\mathrm{N}=237\end{array}$ & HD and PD & Comorbidity (CCI) & HR, CCI score \\
\hline Couchad et al.,[92] 2009 & $\begin{array}{l}\text { French Rein Registry } \\
\qquad \mathrm{N}=2500\end{array}$ & Starting dialysis & Frailty, comorbidity & $\begin{array}{l}\text { OR, Risk Score } \\
0-9\end{array}$ \\
\hline $\begin{array}{l}\text { Cohen et al., }{ }^{[93]} \\
2010\end{array}$ & $\begin{array}{c}\text { Multicenter } \\
\mathrm{N}=449\end{array}$ & HD & $\begin{array}{l}\text { Age, comorbidity, physician's } \\
\text { impression }\end{array}$ & Risk quintiles \\
\hline $\begin{array}{l}\text { van Walraven et al.,[94] } \\
2010\end{array}$ & $\begin{array}{c}\text { USRDS } \\
\mathrm{N}=169,393\end{array}$ & HD, PD, KT & $\begin{array}{c}\text { Age, comorbidity, race, BMI, } \\
\text { listed-year }\end{array}$ & $\begin{array}{l}\text { Increase in risk } \\
\text { score }\end{array}$ \\
\hline $\begin{array}{l}\text { Liu et al.,[95] } \\
2010\end{array}$ & $\begin{array}{l}\text { REMIS, UNOS, } \\
\text { N=244,651 }\end{array}$ & Incident and prevalent & Age, race, comorbidity, CCI & $\begin{array}{c}\text { HR, Increase in } \\
\text { risk score }(\leq 3 \text {, } \\
4-7,7-9, \geq 10)\end{array}$ \\
\hline $\begin{array}{l}\text { Wagner et al.,[96] } \\
2011\end{array}$ & $\begin{array}{l}\text { Multicenter } \\
\mathrm{N}=5447\end{array}$ & $\mathrm{HD}, \mathrm{PD}$ & $\begin{array}{l}\text { Age, race, comorbidity, blood } \\
\text { parameters }\end{array}$ & $\begin{array}{c}\text { Increases in } \\
\text { the HR }\end{array}$ \\
\hline Couchad et al.,[99] 2015 & $\begin{array}{c}\text { REIN } \\
\mathrm{N}=24,348\end{array}$ & Starting dialysis & $\begin{array}{l}\text { Age, gender, frailty, } \\
\text { comorbidity, albumin }\end{array}$ & $\begin{array}{l}\text { OR, Low, } \\
\text { Intermediate, } \\
\text { High }\end{array}$ \\
\hline $\begin{array}{l}\text { Floege et al.,[98] } \\
2015\end{array}$ & $\begin{array}{l}\text { ARO and DOPPS cohorts } \\
\qquad \mathrm{N}=11,508\end{array}$ & Incident HD patients & $\begin{array}{l}\text { Age, comorbidity, vascular } \\
\text { access, blood parameters }\end{array}$ & $\begin{array}{l}\text { Low- } \\
\text { Intermediate- } \\
\text { High risk }\end{array}$ \\
\hline Dusseaux et al.,[100] 2015 & $\begin{array}{c}\text { REIN } \\
N=16,337\end{array}$ & Incident dialysis patients & $\begin{array}{l}\text { Age, gender, comorbidity, } \\
\text { mobility }\end{array}$ & Risk score 6-18 \\
\hline $\begin{array}{l}\text { Reuter et al., }{ }^{[101]} \\
2016\end{array}$ & $\begin{array}{l}\text { Single center } \\
\quad \mathrm{N}=347\end{array}$ & Waitlisted patients & $\begin{array}{c}\text { Procam, Framinghan, ESC-score, } \\
\text { Muenster scores }\end{array}$ & $\begin{array}{l}\text { Increase in } \\
\text { standard } \\
\text { point-scoring } \\
\text { systems }\end{array}$ \\
\hline $\begin{array}{l}\text { Yang, et al.,[103] } \\
2016\end{array}$ & $\begin{array}{c}\text { NHIRD } \\
\mathrm{N}=3046\end{array}$ & HD & $\begin{array}{l}\text { CHADS2, CHA2DS2 and CCI } \\
\text { scores }\end{array}$ & $\begin{array}{l}\text { Risk score, } \\
\text { AUC }\end{array}$ \\
\hline Ivory et al.,[97] 2017 & $\begin{array}{c}\text { Australia-New Zealand } \\
\text { Registry } \\
\mathrm{N}=23,658\end{array}$ & Starting dialysis & Age, comorbidity, late referral & OR, AUC \\
\hline Ma et al.,[102] 2017 & $\begin{array}{l}\text { Single center } \\
\quad \mathrm{N}=461\end{array}$ & Incident PD patients & $\begin{array}{l}\text { CCI, Hemmelgarn score, and Liu } \\
\text { score }\end{array}$ & $\begin{array}{l}\text { Increase in risk } \\
\text { score }\end{array}$ \\
\hline Chen et al., [63] 2017 & $\begin{array}{c}\text { USRDS } \\
\mathrm{N}=159,362\end{array}$ & Incident dialysis patients & $\begin{array}{l}\text { Age, } \mathrm{BMI}<18 \text {, comorbidity and } \\
\text { being institutionalized }\end{array}$ & $\begin{array}{l}\text { Increase in risk } \\
\text { score groups }\end{array}$ \\
\hline
\end{tabular}

Predictors of mortality included age $>80$ years, body mass index $<18 \mathrm{~kg} / \mathrm{m}^{2}$, congestive heart failure, chronic pulmonary disease, immobility and being institutionalized. A scoring system was internally validated, such that 5 -year mortality was $47 \%$ for the lowest risk score group and $>90 \%$ for the highest risk population [63]. The application of this risk score in our two elderly patients is also shown in Table 3. These risk scores could well be used to estimate mortality risk in elderly patients who initiate dialysis, but not all waitlisted patients for KT. Additionally, the prediction capacity could differ substantially depending on specific 


\section{Kidney Blood Pressure Research}

variables or comorbidities included in the models, as recently reported [101-103]. Lastly, a composite risk model has been developed in exclusively waitlisted patients for KT using age, CCI, the presence of a central venous catheter and employment status. Patient survival in patients without risk factors was compared with that in patients with one or more risk factors; the mortality risk increased significantly at each risk level [21]. This could be useful to prioritize selection in high-risk patients waitlisted for KT.

What is the situation in KT recipients? Despite poorer outcomes compared with younger KT recipients, older KT patients show a significant improvement in survival compared with age-matched waitlisted patients. However, death with a functioning graft is still the second most common cause of graft loss, especially in the elderly population [42, 52]. Predicting mortality and comorbidity in these patients could be very convenient to determine the most timely therapeutic measures and thus decrease mortality. Given that application of prognostic indices used in the general population (e.g. Framingham score) could underestimate the mortality risk in the transplant population $[104,105]$, an appropriate assessment of survival in these patients should include both classical and non-classical risk factors plus transplantrelated factors and community-based health indicators in the predictive models, because traditional risk factors alone do not sufficiently explain the high mortality [43]. Finally, frailty has also been recently associated with high mortality in KT recipients $[47,106]$.

Recent years have seen the development and validation of several prognostic indices for mortality and comorbidity (mainly IHD) in KT recipients [12, 32, 40, 55, 63, 94, 107-128] (Table 5) and several e-resources (e.g. www.transplantscore.com, www.transplantmodels. com or www.renalmed.co.uk/risk-calculator) driven from these risk scores are now available for this population [129]. Some of these indices are based on risk scores applied to the general population $[109,111,118,124]$, whereas others have also included pre- and post-transplant variables (Table 5). In general, they all accurately predict the risk of death or appearance of IHD and some attempts have been made to internally validate or compare the performance of these predictive models, mostly using registry data, but as yet, few have been externally validated in prospective studies. The creation of standardized and validated processes for risk model development, using data from national registries, could help transplant teams worldwide and facilitate international comparisons in order to improve transplant outcomes, as previously suggested [130]. Additionally, very few models have exclusively assessed patient survival in advanced-age recipients or they have only focused on the effect of age on survival $[32,40,41,55,63,108,112,116,118]$. As a consequence, there is currently no consensus about the optimal survival prediction risk score for elderly KT. Meanwhile, transplant clinicians should implement the most useful risk model according to their own transplant center or characteristics patients. Table 6 shows several risk scores to predict mortality in KT recipients. These scores have been internally and/or externally validated and they can be used to help transplant physicians in their decision-making in daily clinical practice. For instance, a prognostic index for mortality developed in a single-center cohort study assigned older patients ( $>60$ years) without additional comorbidities to the highestrisk group for death after KT [108], as would be expected in our Patient A (78\% survival at the fifth year post-transplantation), which can be considered as an acceptable mortality and a clinically manageable situation. By contrast, this figure for our Patient B would be $70 \%$ at the same post-transplantation time (Table 3). Grams et al, using a cohort of 6988 KT recipients 65 years or older, developed a prediction model for mortality by logistic regression involving 4 demographic and 15 comorbidity variables [32]. Application of the model to our Patient A gives an acceptable predicted 3-year post-transplant survival (75\%), being considered a priori a good candidate for KT. The predicted 3-year post-transplant survival of our Patient B would be $69 \%$. In a sub-analysis of 67 elderly patients (70 years or older) who received a KT, performed by Dusseux et al. using logistic regression in a cohort of ESRD patients from the French national registry, patients with a risk score $<6$ points showed a good prognosis at the first three years post-transplantation [100]. Using a recent predictive score proposed by Molnar et al. [127], our elderly Patient A would have a probability of death at the first 


\section{Kidney Blood Pressure Research}

Hernández et al.: Mortality in Elderly Waiting-List and Kidney Transplant Patients

Table 5. Predictive models for death and ischemic heart disease in kidney transplantation from deceased or living donors. Abbreviations: ANNs, artificial neural networks; CCI, Charlson Comorbidity Index; CORR, Canadian Organ Replacement Registry; GF, graft failure; HR, hazard ratio; IHD, ischemic heart disease; MACE, major adverse cardiac events, including cardiac death; OPTN, Organ Procurement and Transplantation Network; OR, odds ratio; SIUT, Sindh Institute of Urology and Transplantation database; SRTR, Scientific Registry of Transplant Recipients; UNOS, United Network for Organ Sharing; USRDS, US Renal Data System

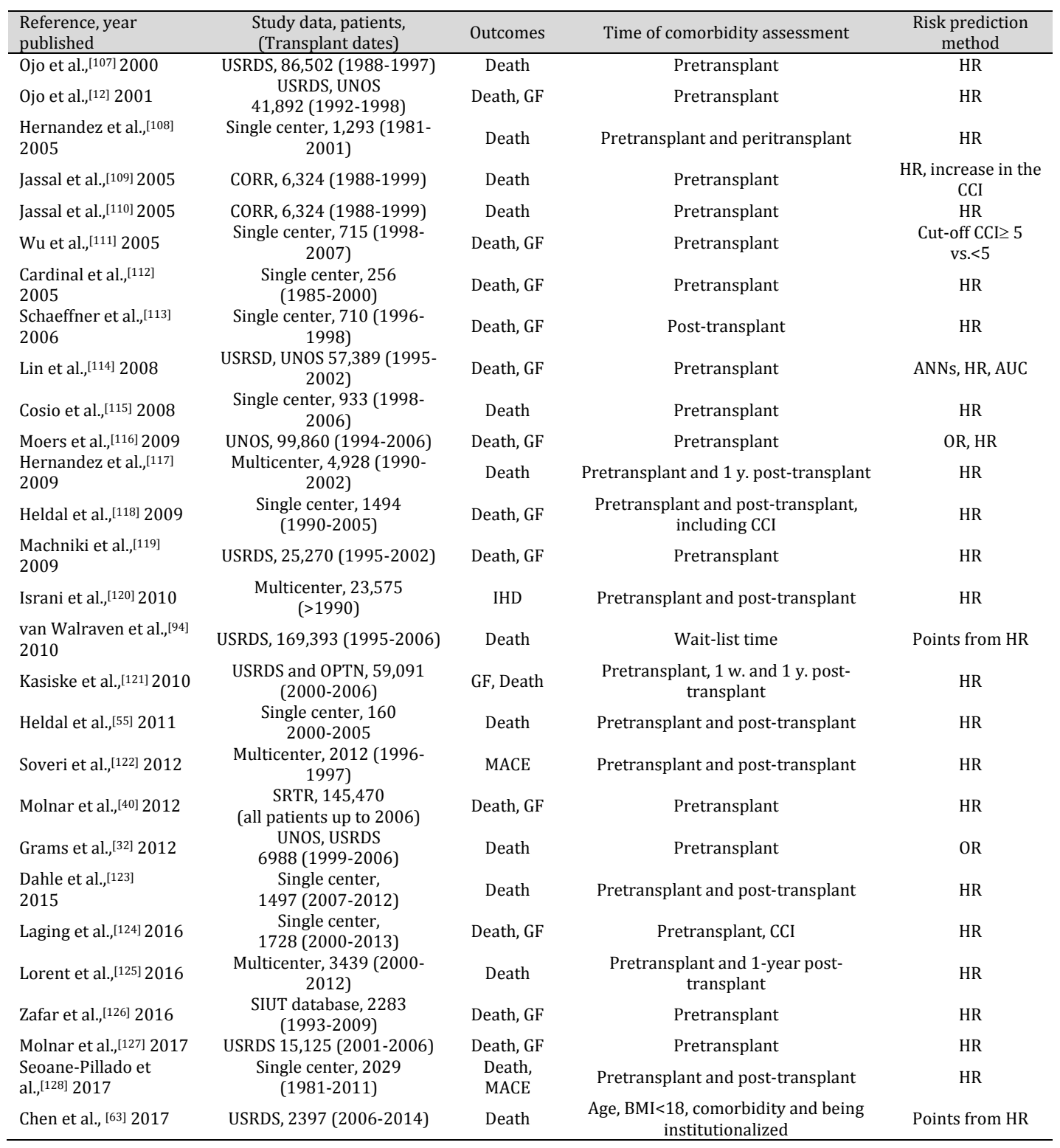

and fifth year post-transplantation of $12 \%$ and $28 \%$, respectively. By contrast, our Patient B would have a higher mortality risk ( $16 \%$ and $50 \%$, respectively), as shown in Table 3 . Lastly, as an alternative to the CCI, Laging et al. developed the Rotterdam Comorbidity in Kidney Transplantation (RoCKeT) score using comorbidities similar to those in the CCI, including age [124]. Not surprisingly, comorbidity was highest (75\%) in the oldest age group (70-79 years). However, $50 \%$ of the patients in the highest comorbidity group survived more than 10 years. Accordingly, the authors concluded that patients with severe comorbidity should not, a priori, be excluded from KT because transplant offers better survival than dialysis therapy. Here, the 5-year post-transplant mortality in our Patient A would be 6\%, whereas it would be $30 \%$ for our Patient B (Table 3). When the risk scoring system elaborated by 


\section{Kidney \\ Blood Pressure Research}

Table 6. Several published scores, internally and/or externally validated in other populations, to predict mortality after kidney transplant. Abbreviations: CCI, comorbidity Charlson Index; TX, kidney transplant, CVD, cardiovascular disease; WL, waiting list; PVD, peripheral vascular disease; CVA, cerebrovascular accident; HIV, human immunodeficiency virus; RRS, Recipient Risk Score; MACE, major adverse cardiovascular events

\begin{tabular}{|c|c|c|}
\hline $\begin{array}{l}\text { Reference, year published, } \\
\text { (transplant dates) }\end{array}$ & Population & Assessment/risk stratification \\
\hline $\begin{array}{l}\text { Wu et al., [111] } 2005 \\
(1998-2003)\end{array}$ & All ages (>18 y) & $\begin{array}{l}\text { Assessment: cut-off of pretransplant CCI score }(\mathrm{CCI}<5 \text { vs. } \geq 5 \text { ) } \\
\qquad \quad \text { Scoring based on: age and CCI } \\
\text { Assessment: Patients were stratified into three risk groups (low, medium, } \\
\text { and high) by combining peritransplant risk factors for mortality after }\end{array}$ \\
\hline $\begin{array}{l}\text { Hernandez et al., [108] } 2005 \\
(1981-2001)\end{array}$ & All ages (>18 y) & $\begin{array}{l}\text { hospitalization and using beta-coefficient from multivariate Cox analysis } \\
\text { - Scoring based on: age, selected pretransplant comorbidities, renal } \\
\text { dysfunction at discharge, diabetes, time on dialysis, and acute tubular } \\
\text { necrosis }\end{array}$ \\
\hline $\begin{array}{l}\text { Cardinal et al., }{ }^{[112]} 2005 \\
(1985-2000)\end{array}$ & $\geq 60$ ys. & $\begin{array}{l}\text { Assessment: Relative risk of mortality and crude rates of patient survival at } \\
\qquad 1,5 \text {, and } 10 \text { years post-transplant } \\
\text { Scoring based on: smoking, BMI, time on dialysis before TX }\end{array}$ \\
\hline $\begin{array}{l}\text { Hernandez et al., [117] } 2009 \\
(1990-2002)\end{array}$ & All ages $(>18 \mathrm{y})$ & $\begin{array}{l}\text { Assessment: The probability of } 3 \text {-year post-transplant mortality by score } \\
\text { risk quartiles, using beta-coefficient from multivariate Cox analysis } \\
\text { - Scoring based on: age, hepatitis C, diabetes (Pre and post-TX), } \\
\text { proteinuria and renal function at the } 1^{\text {st }} \mathrm{y} \text {, and immunosuppressants }\end{array}$ \\
\hline $\begin{array}{l}\text { Machnicki et al., [119] } 2009 \\
(1995-2002)\end{array}$ & All ages $(>18 y)$ & $\begin{array}{l}\text { Assessment: Adjusted hazard risks for death using multivariate Cox } \\
\text { regression models } \\
\text { - Score based on: pretransplant comorbidities from Medicare claims with } \\
\text { the Clinical Classifications Software (CCS), Charlson and Elixhauser } \\
\text { comorbidities }\end{array}$ \\
\hline $\begin{array}{l}\text { van Walraven et al., [94] } 2010 \\
(1995-2006)\end{array}$ & All ages (>18 y) & $\begin{array}{l}\text { Assessment: Scoring system: An individual patient's index score can be } \\
\text { calculated by summing up the points for each applicable risk factor } \\
\text { - Scoring based on: age stratus, BMI, cause of renal disease, race, year of } \\
\text { transplant, comorbidities and time on WL }\end{array}$ \\
\hline $\begin{array}{l}\text { Kasiske et al., }{ }^{[121]} 2010 \\
(2000-2006)\end{array}$ & All ages (>18 y) & $\begin{array}{l}\text { Assessment: The final prediction models can be used to estimate predicted } \\
\text { probability of both all-cause mortality and graft failure within } 5 \text { years of } \\
\text { transplant from each of } 3 \text { times: at the time of transplant, } 7 \text { days post- } \\
\text { transplant, and } 1 \text { year post-transplant } \\
\text { - Scoring based on: pretransplant, } 1 \text { st week and 1-year post-transplant } \\
\text { clinical variables }\end{array}$ \\
\hline $\begin{array}{l}\text { Soveri et al., }{ }^{122]} 2012 \\
\text { (1996-1997) } \\
\text { Cardiovascular Risk Calculator } \\
\text { for Renal Transplant Recipients, } \\
\text { http://www.anst.uu.se/ } \\
\text { insov254/calculator/ }\end{array}$ & All ages (>18 y) & $\begin{array}{l}\text { Assessment: MACE-Free Survival Calculation (probability of 7-year MACE- } \\
\text { free survival) } \\
\text { - Scoring based on: recipient age, smoking, coronary heart disease, } \\
\text { diabetes, creatinine, renal replacement therapy and number of } \\
\text { transplants }\end{array}$ \\
\hline $\begin{array}{l}\text { Grams et al., [32] } 2012 \\
(1999-2006)\end{array}$ & $\geq 65 \mathrm{y}$ & $\begin{array}{l}\text { Assessment: quintile of predicted 3-year post-transplantation survival (top } \\
\text { quintile, excellent candidates; quintiles } 2 \text { and 3, good candidates; quintiles } 4 \\
\text { and 5, remaining candidates. } \\
\text { - Scoring based on: age recipient, time on dialysis, drug-depent, selected } \\
\text { comorbidities, smoking and transplant year }>1999\end{array}$ \\
\hline $\begin{array}{l}\text { Sorensen et al., }{ }^{[17]} 2016 \\
(1995-2011)\end{array}$ & All ages $(>18 y)$ & $\begin{array}{l}\text { Assessment: Mortality risk combining recipient age, type of transplant and } \\
\qquad \text { CCI }(<2,3-4 \text { and } \geq 5) \\
\text { - Scoring based on: recipient age (five stratus), type of transplant and CCI } \\
\text { Assessment: two comorbidity scores: The Rotterdam Comorbidity in Kidney }\end{array}$ \\
\hline $\begin{array}{l}\text { Laging et al., [124] } 2016 \\
(2000-2013)\end{array}$ & All ages $(>18 y)$ & $\begin{array}{c}\text { Transplantation score (four strati: } 0,1-2,3-4,5-9 \text { ) and CCI } \\
\text { - Scoring based on: age, diabetes, malignancy. HIV, PVD, CVA, liver } \\
\text { disease and lung disease or CCI }\end{array}$ \\
\hline $\begin{array}{l}\text { Lorent et al., }[125] 2016 \\
\text { (two cohorts: } 2000-2012 \text { and } \\
\text { 2008-2012) }\end{array}$ & All ages $(>18 y)$ & $\begin{array}{l}\text { Assessment: 1-year mortality scoring system: cut-off at } 0.03 \text { for the } 1 \text {-year } \\
\text { RRS and } 2.24 \text { for the RRS } \\
\text { - Scoring based on: recipient age, time on dialysis, pretransplant CVD and } \\
\text { 1-year serum creatinine or RRS }\end{array}$ \\
\hline $\begin{array}{l}\text { Molnar et al., [127] } 2017 \text { (2001- } \\
\text { 2006) } \\
\text { www.TransplantScore.com }\end{array}$ & $>65 y$ & $\begin{array}{l}\text { Assessment: estimated post-transplant survival using beta-coefficients from } \\
\text { multivariable model for mortality } \\
\text { - Scoring based on: recipient age, race, cause and length of renal disease, } \\
\text { biochemical parameters, selected comorbidities, donor characteristics, } \\
\text { diabetes status and type of insurance }\end{array}$ \\
\hline Chen et al., [63] 2017 (2006-2013) & $>70 y$ & $\begin{array}{c}\text { Assessment: scoring system: } 1 \text { (low risk) to } 5 \text { (high risk) points } \\
\text { - Scoring based on: recipient age }>80 \text { y; BMI<18; comorbidities; } \\
\text { immobilization; institutionalized patients }\end{array}$ \\
\hline
\end{tabular}

Chen at al for elderly dialysis patients was applied to KT recipients, the 5-year mortality was approximately $40 \%$ in the highest risk score, whereas it was $90 \%$ for age-matched high risk candidates for KT [63]. The fact that use of expanded criteria donor kidneys has not 


\section{Kidney \\ Blood Pressure Research}

been associated with increased mortality in recipients older than 70 years supports these findings [40].

\section{Conclusion}

Advanced age should not be an absolute contraindication to KT in carefully selected ESRD patients, because KT offers a higher survival rate than dialysis therapy, even in the elderly. Waitlisted patients, especially older patients, have a higher mortality risk than agematched KT recipients. Thus, identification of risk factors for death in waitlisted patients, especially elderly candidates, could help to allocate and prioritize high-risk patients for KT. As the number of organs is limited worldwide, decisions should be based on the best clinical evidence to afford equal opportunities of receiving KT in both high-risk and low-risk waitlisted elderly patients. Finally, older KT recipients have a significantly higher mortality, mainly those receiving low-quality grafts, than younger KT patients. Thus, risk scores to predict both perioperative and early post-transplant mortality could contribute to the better management of high-risk patients and the use of prophylactic interventions to prolong survival, provided a higher mortality is assumed.

\section{Disclosure Statement}

The authors declare they have no conflicts of interest regarding the publication of this article.

\section{Abbreviations}

ASA (American Society of Anesthesiology); BMI (body mass index); CCI (Charlson Comorbidity index); CV (cardiovascular); ESRD (end-stage renal disease); IHD (ischemic heart disease); KT (kidney transplantation); PVD (peripheral vascular disease); QOL (quality of life); WL (waiting list).

\section{Acknowledgements}

This study was supported in partby the Spanish Ministry of Economy and Competitiveness (MINECO) (grant ICI14/00016) from the Instituto de Salud Carlos III co-funded by the Fondo Europeo de Desarrollo Regional-FEDER, RETICS (REDINREN RD12/0021/0015, RD16/0009/0006, RD16/0009/0031). The authors thank the Andalusian Transplant Coordinator Center as well as the transplant teams and all participants in each center for their collaboration. We also thank Ian Johnstone for linguistic assistance in the preparation of the text.

\section{References}

1 Saran R, Robinson B, Abbott KC, Agodoa LYC, Albertus P, Ayanian J, Balkrishnan R, Bragg-Gresham J, Cao J, Chen JLT, Cope E, Dharmarajan S, Dietrich X, Eckard A, Eggers PW, Gaber C, Gillen D, Gipson D, Gu H, Hailpern SM, et al.: US Renal Data System 2016 Annual Data Report: Epidemiology of Kidney Disease in the United States. Am J Kidney Dis 2017;69:A7-A8. 


\section{Kidney \\ Blood Pressure Research}

Hernández et al.: Mortality in Elderly Waiting-List and Kidney Transplant Patients

2 Pippias M, Stel VS, Abad Diez JM, Afentakis N, Herrero-Calvo JA, Arias M, Tomilina N, Bouzas Caamaño E, Buturovic-Ponikvar J, Čala S, Caskey FJ, Castro de la Nuez P, Cernevskis H, Collart F, Alonso de la Torre R, García Bazaga M de LÁ, De Meester J, Díaz JM, Djukanovic L, Ferrer Alamar M, et al.: Renal replacement therapy in Europe: a summary of the 2012 ERA-EDTA Registry Annual Report. Clin Kidney J 2015;8:248261.

3 Sørensen SS: Rates of renal transplantations in the elderly-data from Europe and the US. Transplant Rev (Orlando) 2015;29:193-196.

-4 Segall L, Nistor I, Pascual J, Mucsi I, Guirado L, Higgins R, Van Laecke S, Oberbauer R, Van Biesen W, Abramowicz D, Gavrilovici C, Farrington K, Covic A: Criteria for and Appropriateness of Renal Transplantation in Elderly Patients With End-Stage Renal Disease: A Literature Review and Position Statement on Behalf of the European Renal Association-European Dialysis and Transplant Association Descartes Working Group and European Renal Best Practice. Transplantation 2016;100:e55-65.

>5 van der Veer SN, van Biesen W, Bernaert P, Bolignano D, Brown EA, Covic A, Farrington K, Jager KJ, Kooman J, Macías-Núñez JF, Mooney A, van Munster BC, Topinkova E, Van Den Noortgate NJA, Wirnsberger G, Michel J-P, Nistor I: Priority topics for European multidisciplinary guidelines on the management of chronic kidney disease in older adults. Int Urol Nephrol 2016;48:859-869.

-6 European Renal Best Practice Transplantation Guideline Development Group: ERBP Guideline on the Management and Evaluation of the Kidney Donor and Recipient. Nephrol Dial Transplant 2013;28:ii1-71.

-7 Abramowicz D, Cochat P, Claas FHJ, Heemann U, Pascual J, Dudley C, Harden P, Hourmant M, Maggiore U, Salvadori M, Spasovski G, Squifflet J-P, Steiger J, Torres A, Viklicky O, Zeier M, Vanholder R, Van Biesen W, Nagler E: European Renal Best Practice Guideline on kidney donor and recipient evaluation and perioperative care. Nephrol Dial Transplant 2015;30:1790-1797.

-8 Montero N, Pérez-Sáez MJ, Pascual J, DESCARTES Working Group, DESCARTES ERA-EDTA Board:, Abramowicz D, Budde K, Dudley C, Hazzan M, Klinger M, Maggiore U, Oberbauer R, Pascual J, Sorensen SS, Viklicky O: Immunosuppression in the elderly renal allograft recipient: a systematic review. Transplant Rev (Orlando) 2016;30:144-153.

9 Ponticelli C, Podestà MA, Graziani G: Renal transplantation in elderly patients. How to select the candidates to the waiting list? Transplant Rev (Orlando) 2014;28:188-192.

10 Schnuelle P, Lorenz D, Trede M, Van Der Woude FJ: Impact of renal cadaveric transplantation on survival in end-stage renal failure: evidence for reduced mortality risk compared with hemodialysis during long-term follow-up. J Am Soc Nephrol 1998;9:2135-2141.

11 Wolfe RA, Ashby VB, Milford EL, Ojo AO, Ettenger RE, Agodoa LYC, Held PJ, Port FK: Comparison of mortality in all patients on dialysis, patients on dialysis awaiting transplantation, and recipients of a first cadaveric transplant. N Engl J Med 1999;341:1725-1730.

-12 Ojo AO, Hanson JA, Meier-Kriesche H, Okechukwu CN, Wolfe RA, Leichtman AB, Agodoa LY, Kaplan B, Port FK: Survival in recipients of marginal cadaveric donor kidneys compared with other recipients and waitlisted transplant candidates. J Am Soc Nephrol 2001;12:589-597.

13 Rao PS, Merion RM, Ashby VB, Port FK, Wolfe RA, Kayler LK: Renal transplantation in elderly patients older than 70 years of age: results from the Scientific Registry of Transplant Recipients. Transplantation 2007;83:1069-1074.

14 Gill JS, Rose C, Pereira BJG, Tonelli M: The importance of transitions between dialysis and transplantation in the care of end-stage renal disease patients. Kidney Int 2007;71:442-447.

-15 Hod T, Goldfarb-Rumyantzev AS: Clinical issues in renal transplantation in the elderly. Clin Transplant 2015;29:167-175.

-16 Jassal S V, Krahn MD, Naglie G, Zaltzman JS, Roscoe JM, Cole EH, Redelmeier DA: Kidney transplantation in the elderly: a decision analysis. J Am Soc Nephrol 2003;14:187-196.

17 Sørensen VR, Heaf J, Wehberg S, Sørensen SS: Survival Benefit in Renal Transplantation Despite High Comorbidity. Transplantation 2016;100:2160-2167.

18 de Fijter JW: An old virtue to improve senior programs. Transpl Int 2009;22:259-268.

19 Schold JD, Meier-Kriesche HU: Which renal transplant candidates should accept marginal kidneys in exchange for a shorter waiting time on dialysis? Clin J Am Soc Nephrol 2006;1:532-538.

-20 Delmonico FL, McBride MA: Analysis of the wait list and deaths among candidates waiting for a kidney transplant. Transplantation 2008;86:1678-1683. 


\section{Kidney \\ Blood Pressure Research}

-21 Hernández D, De La Nuez PC, Muriel A, Ruiz-Esteban P, González-Molina M, Burgos D, Cabello M, Luengo S, Palma E, Ruda E, Gutiérrez E, Vozmediano C, Torres A, Alonso M: Clinical assessment of mortality risk in renal transplant candidates in Spain. Transplantation 2014;98:653-659.

-22 Schold J, Srinivas TR, Sehgal AR, Meier-Kriesche HU: Half of kidney transplant candidates who are older than 60 years now placed on the waiting list will die before receiving a deceased-donor transplant. Clin J Am Soc Nephrol 2009;4:1239-1245.

-23 Matas AJ, Smith JM, Skeans MA, Thompson B, Gustafson SK, Stewart DE, Cherikh WS, Wainright JL, Boyle G, Snyder JJ, Israni AK, Kasiske BL: OPTN/SRTR 2013 Annual Data Report: kidney. Am J Transplant 2015;15:1-34.

-24 Schold JD, Srinivas TR, Kayler LK, Meier-Kriesche HU: The overlapping risk profile between dialysis patients listed and not listed for renal transplantation. Am J Transplant 2008;8:58-68.

-25 Lynch RJ, Zhang R, Patzer RE, Larsen CP, Adams AB: First-Year Waitlist Hospitalization and Subsequent Waitlist and Transplant Outcome. Am J Transplant 2017;17:1031-1041.

26 Bayat S, Kessler M, Briançon S, Frimat L: Survival of transplanted and dialysed patients in a French region with focus on outcomes in the elderly. Nephrol Dial Transplant 2010;25:292-300.

27 Charlson ME, Pompei P, Ales KL, MacKenzie CR: A new method of classifying prognostic comorbidity in longitudinal studies: development and validation. J Chronic Dis 1987;40:373-383.

28 Snyder JJ, Kasiske BL, Maclean R: Peripheral arterial disease and renal transplantation. J Am Soc Nephrol 2006;17:2056-2068.

29 Liu T, Liang K V, Rosenbaum A, Stephenson R, Pike F, Weissfeld L, Unruh ML: Peripheral vascular disease severity impacts health outcomes and health-related quality of life in maintenance hemodialysis patients in the HEMO Study. Nephrol Dial Transplant 2012;27:2929-2936.

-30 Hernández D, De La Nuez PC, Muriel A, Ruiz-Esteban P, Rudas E, González-Molina M, Burgos D, Cabello M, Palma E, Gutiérrez E, Alonso M: Peripheral vascular disease \&amp; death in southern european kidney transplant candidates: A competing risk modeling approach. Transplantation 2017;101:1320-1326.

-31 Schold JD, Heaphy ELG, Buccini LD, Poggio ED, Srinivas TR, Goldfarb DA, Flechner SM, Rodrigue JR, Thornton JD, Sehgal AR: Prominent impact of community risk factors on kidney transplant candidate processes and outcomes. Am J Transplant 2013;13:2374-2383.

-32 Grams ME, Kucirka LM, Hanrahan CF, Montgomery RA, Massie AB, Segev DL: Candidacy for kidney transplantation of older adults. J Am Geriatr Soc 2012;60:1-7.

33 Ng YY, Hung YN, Wu SC, Ko PJ, Hwang SM: Progression in comorbidity before hemodialysis initiation is a valuable predictor of survival in incident patients. Nephrol Dial Transplant 2013;28:1005-1012.

34 Couchoud C, Bayat S, Villar E, Jacquelinet C, Ecochard R, REIN registry: A new approach for measuring gender disparity in access to renal transplantation waiting lists. Transplantation 2012;94:513-519.

-35 Khattak MW, Sandhu GS, Woodward R, Stoff JS, Goldfarb-Rumyantzev AS: Association of marital status with access to renal transplantation. Am J Transplant 2010;10:2624-2631.

-36 Gill JS, Hendren E, Dong J, Johnston 0, Gill J: Differential association of body mass index with access to kidney transplantation in men and women. Clin J Am Soc Nephrol 2014;9:951-959.

-37 Meier-Kriesche HU, Ojo AO, Hanson JA, Kaplan B: Exponentially increased risk of infectious death in older renal transplant recipients. Kidney Int 2001;59:1539-1543.

38 Meier-Kriesche HU, Ojo A, Hanson J, Cibrik D, Lake K, Agodoa LY, Leichtman A, Kaplan B: Increased immunosuppressive vulnerability in elderly renal transplant recipients. Transplantation 2000;69:885-889.

-39 Kauffman HM, McBride MA, Cors CS, Roza AM, Wynn JJ: Early mortality rates in older kidney recipients with comorbid risk factors. Transplantation 2007;83:404-410.

40 Molnar MZ, Streja E, Kovesdy CP, Shah A, Huang E, Bunnapradist S, Krishnan M, Kopple JD, Kalantar-Zadeh K: Age and the associations of living donor and expanded criteria donor kidneys with kidney transplant outcomes. Am J Kidney Dis 2012;59:841-848.

41 Iordanous Y, Seymour N, Young A, Johnson J, Iansavichus A V, Cuerden MS, Gill JS, Poggio E, Garg AX, Donor Nephrectomy Outcomes Research (DONOR) Network: Recipient outcomes for expanded criteria living kidney donors: the disconnect between current evidence and practice. Am J Transplant 2009;9:1558-1573.

-42 Hernández D, Moreso F: Has patient survival following renal transplantation improved in the era of modern immunosuppression? Nefrologia 2013;33:171-180. 


\section{Kidney \\ Blood Pressure Research}

43 Hernández D, Muriel A, Abraira V: Current state of clinical end-points assessment in transplant: Key points. Transplant Rev (Orlando) 2016;30:92-99.

44 Knoll GA: Kidney transplantation in the older adult. Am J Kidney Dis 2013;61:790-797.

45 Karim A, Farrugia D, Cheshire J, Mahboob S, Begaj I, Ray D, Sharif A: Recipient age and risk for mortality after kidney transplantation in England. Transplantation 2014;97:832-838.

46 Pinter J, Hanson CS, Chapman JR, Wong G, Craig JC, Schell JO, Tong A: Perspectives of Older Kidney Transplant Recipients on Kidney Transplantation. Clin J Am Soc Nephrol 2017;12:443-453.

47 McAdams-DeMarco MA, Bae S, Chu N, Gross AL, Brown CH, Oh E, Rosenberg P, Neufeld KJ, Varadhan R, Albert M, Walston J, Segev DL: Dementia and Alzheimer's Disease among Older Kidney Transplant Recipients. J Am Soc Nephrol 2017;28:1575-1583.

-48 King EA, Bowring MG, Massie AB, Kucirka LM, McAdams-DeMarco MA, Al-Ammary F, Desai NM, Segev DL: Mortality and Graft Loss Attributable to Readmission following Kidney Transplantation: Immediate and Long-Term Risk. Transplantation 2017. DOI: 10.1097/TP.0000000000001609.

49 McAdams-DeMarco MA, Ying H, Olorundare I, King EA, Haugen C, Buta B, Gross AL, Kalyani R, Desai NM, Dagher NN, Lonze BE, Montgomery RA, Bandeen-Roche K, Walston JD, Segev DL: Individual Frailty Components and Mortality in Kidney Transplant Recipients. Transplantation 2017;101:2126-2132.

50 Reese PP, Shults J, Bloom RD, Mussell A, Harhay MN, Abt P, Levine M, Johansen KL, Karlawish JT, Feldman HI: Functional status, time to transplantation, and survival benefit of kidney transplantation among waitlisted candidates. Am J Kidney Dis 2015;66:837-845.

51 Keith DS, Cantarovich M, Paraskevas S, Tchervenkov J: Recipient age and risk of chronic allograft nephropathy in primary deceased donor kidney transplant. Transpl Int 2006;19:649-656.

-52 Huang E, Poommipanit N, Sampaio MS, Kuo H-T, Reddy P, Gritsch HA, Pham P-T, Wilkinson A, Danovitch G, Bunnapradist S: Intermediate-term outcomes associated with kidney transplantation in recipients 80 years and older: an analysis of the OPTN/UNOS database. Transplantation 2010;90:974-979.

-53 Wong G, Howard K, Chapman JR, Chadban S, Cross N, Tong A, Webster AC, Craig JC: Comparative survival and economic benefits of deceased donor kidney transplantation and dialysis in people with varying ages and co-morbidities. PLoS One 2012;7:e29591.

54 Lønning K, Midtvedt K, Leivestad T, Reisæter A V, Line P-D, Hartmann A, Heldal K: Are Octogenarians With End-Stage Renal Disease Candidates for Renal Transplantation? Transplantation 2016;100:2705-2709.

55 Heldal K, Hartmann A, Leivestad T, Foss A, Midtvedt K: Risk variables associated with the outcome of kidney recipients \&gt;70 years of age in the new millennium. Nephrol Dial Transplant 2011;26:2706-2711.

56 Heldal K, Hartmann A, Grootendorst DC, de Jager DJ, Leivestad T, Foss A, Midtvedt K: Benefit of kidney transplantation beyond 70 years of age. Nephrol Dial Transplant 2010;25:1680-1687.

57 Oniscu GC, Schalkwijk AAH, Johnson RJ, Brown H, Forsythe JLR: Equity of access to renal transplant waiting list and renal transplantation in Scotland: cohort study. BMJ 2003;327:1261.

58 Savoye E, Tamarelle D, Chalem Y, Rebibou J-M, Tuppin P: Survival benefits of kidney transplantation with expanded criteria deceased donors in patients aged 60 years and over. Transplantation 2007;84:16181624.

-59 Johnson DW, Herzig K, Purdie D, Brown AM, Rigby RJ, Nicol DL, Hawley CM: A comparison of the effects of dialysis and renal transplantation on the survival of older uremic patients. Transplantation 2000;69:794799.

60 Molnar MZ, Ravel V, Streja E, Kovesdy CP, Rivara MB, Mehrotra R, Kalantar-Zadeh K: Survival of Elderly Adults Undergoing Incident Home Hemodialysis and Kidney Transplantation. J Am Geriatr Soc 2016;64:2003-2010.

61 Hellemans R, Stel VS, Jager KJ, Bosmans JL, Abramowicz D: Do elderly recipients really benefit from kidney transplantation? Transplant Rev (Orlando) 2015;29:197-201.

62 Goodkin DA, Young EW, Kurokawa K, Prütz KG, Levin NW: Mortality among hemodialysis patients in Europe, Japan, and the United States: case-mix effects. Am J Kidney Dis 2004;44:16-21.

63 Chen L-X, Josephson MA, Hedeker D, Campbell KH, Stankus N, Saunders MR: A Clinical Prediction Score to Guide Referral of Elderly Dialysis Patients for Kidney Transplant Evaluation. Kidney Int reports 2017;2:645-653.

64 Jay CL, Washburn K, Dean PG, Helmick RA, Pugh JA, Stegall MD: Survival Benefit in Older Patients Associated With Earlier Transplant With High KDPI Kidneys. Transplantation 2017;101:867-872. 


\section{Kidney \\ Blood Pressure Research}

\begin{tabular}{l}
\hline Kidney Blood Press Res 2018;43:256-275 \\
\hline \begin{tabular}{l|l} 
DOI: 10.1159/000487684 & (c) 2018 The Author(s). Published by S. Karger AG, Basel \\
Published onlIne: February 27, 2018 & www.karger.com/kbr
\end{tabular}
\end{tabular}

65 Cohen JB, Eddinger KC, Locke JE, Forde KA, Reese PP, Sawinski DL: Survival Benefit of Transplantation with a Deceased Diabetic Donor Kidney Compared with Remaining on the Waitlist. Clin J Am Soc Nephrol 2017;12:974-982.

66 Peters-Sengers H, Berger SP, Heemskerk MBA, al Arashi D, Homan van der Heide JJ, Hemke AC, ten Berge IJM, Idu MM, Betjes MGH, van Zuilen AD, Hilbrands LB, de Vries APJ, Nurmohamed AS, Christiaans MH, Ernest van Heurn LW, de Fijter JW, Bemelman FJ: Stretching the Limits of Renal Transplantation in Elderly Recipients of Grafts from Elderly Deceased Donors. J Am Soc Nephrol 2017;28:621-631.

67 Tonelli M, Wiebe N, Knoll G, Bello A, Browne S, Jadhav D, Klarenbach S, Gill J: Systematic review: kidney transplantation compared with dialysis in clinically relevant outcomes. Am J Transplant 2011;11:20932109.

68 Pérez-Sáez MJ, Arcos E, Comas J, Crespo M, Lloveras J, Pascual J, Catalan Renal Registry Committee: Survival Benefit From Kidney Transplantation Using Kidneys From Deceased Donors Aged $\geq 75$ Years: A TimeDependent Analysis. Am J Transplant 2016;16:2724-2733.

-69 Pérez-Sáez MJ, Montero N, Redondo-Pachón D, Crespo M, Pascual J: Strategies for an Expanded Use of Kidneys From Elderly Donors. Transplantation 2017;101:727-745.

70 0jo A0: Cardiovascular complications after renal transplantation and their prevention. Transplantation 2006;82:603-611.

71 Gill JS, Schaeffner E, Chadban S, Dong J, Rose C, Johnston 0, Gill J: Quantification of the early risk of death in elderly kidney transplant recipients. Am J Transplant 2013;13:427-432.

72 Batabyal P, Chapman JR, Wong G, Craig JC, Tong A: Clinical practice guidelines on wait-listing for kidney transplantation: consistent and equitable? Transplantation 2012;94:703-713.

73 Fitz-Henry J: The ASA classification and peri-operative risk. Ann R Coll Surg Engl 2011;93:185-187.

74 Lee TH, Marcantonio ER, Mangione CM, Thomas EJ, Polanczyk CA, Cook EF, Sugarbaker DJ, Donaldson MC, Poss R, Ho KK, Ludwig LE, Pedan A, Goldman L: Derivation and prospective validation of a simple index for prediction of cardiac risk of major noncardiac surgery. Circulation 1999;100:1043-1049.

-75 Winoker JS, Paulucci DJ, Anastos H, Waingankar N, Abaza R, Eun DD, Bhandari A, Hemal AK, Sfakianos JP, Badani KK: Predicting Complications Following Robot-Assisted Partial Nephrectomy with the ACS NSQIP(®) Universal Surgical Risk Calculator. J Urol 2017;198:803-809.

76 Gupta PK, Gupta H, Sundaram A, Kaushik M, Fang X, Miller WJ, Esterbrooks DJ, Hunter CB, Pipinos II, Johanning JM, Lynch TG, Forse RA, Mohiuddin SM, Mooss AN: Development and validation of a risk calculator for prediction of cardiac risk after surgery. Circulation 2011;124:381-387.

77 Detsky AS, Abrams HB, McLaughlin JR, Drucker DJ, Sasson Z, Johnston N, Scott JG, Forbath N, Hilliard JR: Predicting cardiac complications in patients undergoing non-cardiac surgery. J Gen Intern Med;1:211-219.

78 Protopapa KL, Simpson JC, Smith NCE, Moonesinghe SR: Development and validation of the Surgical Outcome Risk Tool (SORT). Br J Surg 2014;101:1774-1783.

-79 Van Manen JG, Korevaar JC, Dekker FW, Boeschoten EW, Bossuyt PMM, Krediet RT, NECOSAD-Study Group: Adjustment for comorbidity in studies on health status in ESRD patients: which comorbidity index to use? J Am Soc Nephrol 2003;14:478-485.

-80 Ene-Iordache B, Perico N, Bikbov B, Carminati S, Remuzzi A, Perna A, Islam N, Bravo RF, Aleckovic-Halilovic M, Zou H, Zhang L, Gouda Z, Tchokhonelidze I, Abraham G, Mahdavi-Mazdeh M, Gallieni M, Codreanu I, Togtokh A, Sharma SK, Koirala P, Uprety S, Ulasi I, Remuzzi G: Chronic kidney disease and cardiovascular risk in six regions of the world (ISN-KDDC): a cross-sectional study. Lancet Glob Heal 2016;4:e307-e319.

81 Hutchinson TA, Thomas DC, MacGibbon B: Predicting survival in adults with end-stage renal disease: an age equivalence index. Ann Intern Med 1982;96:417-423.

82 Wright LF: Survival in patients with end-stage renal disease. Am J Kidney Dis 1991;17:25-28.

-83 Khan IH, Catto GR, Edward N, Fleming LW, Henderson IS, MacLeod AM: Influence of coexisting disease on survival on renal-replacement therapy. Lancet (London, England) 1993;341:415-418.

84 Davies SJ, Russell L, Bryan J, Phillips L, Russell GI: Comorbidity, urea kinetics, and appetite in continuous ambulatory peritoneal dialysis patients: their interrelationship and prediction of survival. Am J Kidney Dis 1995;26:353-361.

85 Barrett BJ, Parfrey PS, Morgan J, Barré P, Fine A, Goldstein MB, Handa SP, Jindal KK, Kjellstrand CM, Levin A, Mandin H, Muirhead N, Richardson RM: Prediction of early death in end-stage renal disease patients starting dialysis. Am J Kidney Dis 1997;29:214-222. 


\section{Kidney \\ Blood Pressure Research}

-86 Fried L, Bernardini J, Piraino B: Charlson comorbidity index as a predictor of outcomes in incident peritoneal dialysis patients. Am J Kidney Dis 2001;37:337-342.

87 Beddhu S, Zeidel ML, Saul M, Seddon P, Samore MH, Stoddard GJ, Bruns FJ: The effects of comorbid conditions on the outcomes of patients undergoing peritoneal dialysis. Am J Med 2002;112:696-701.

-88 van Manen JG, Korevaar JC, Dekker FW, Boeschoten EW, Bossuyt PMM, Krediet RT, NECOSAD Study Group. Netherlands Co-operative Study on the Adequacy of Dialysis-2: How to adjust for comorbidity in survival studies in ESRD patients: a comparison of different indices. Am J Kidney Dis 2002;40:82-89.

89 Miskulin DC, Meyer KB, Martin AA, Fink NE, Coresh J, Powe NR, Klag MJ, Levey AS, Choices for Healthy Outcomes in Caring for End-Stage Renal Disease (CHOICE) Study: Comorbidity and its change predict survival in incident dialysis patients. Am J Kidney Dis 2003;41:149-161.

90 Miskulin DC, Martin AA, Brown R, Fink NE, Coresh J, Powe NR, Zager PG, Meyer KB, Levey AS, Medical Directors, Dialysis Clinic, Inc: Predicting 1 year mortality in an outpatient haemodialysis population: a comparison of comorbidity instruments. Nephrol Dial Transplant 2004;19:413-420.

-91 Hemmelgarn BR, Manns BJ, Quan H, Ghali WA: Adapting the Charlson Comorbidity Index for use in patients with ESRD. Am J Kidney Dis 2003;42:125-132.

-92 Couchoud C, Labeeuw M, Moranne O, Allot V, Esnault V, Frimat L, Stengel B, French Renal Epidemiology and Information Network (REIN) registry: A clinical score to predict 6-month prognosis in elderly patients starting dialysis for end-stage renal disease. Nephrol Dial Transplant 2009;24:1553-1561.

93 Cohen LM, Ruthazer R, Moss AH, Germain MJ: Predicting six-month mortality for patients who are on maintenance hemodialysis. Clin J Am Soc Nephrol 2010;5:72-79.

-94 van Walraven C, Austin PC, Knoll G: Predicting potential survival benefit of renal transplantation in patients with chronic kidney disease. CMAJ 2010;182:666-672.

-95 Liu J, Huang Z, Gilbertson DT, Foley RN, Collins AJ: An improved comorbidity index for outcome analyses among dialysis patients. Kidney Int 2010;77:141-151.

-96 Wagner M, Ansell D, Kent DM, Griffith JL, Naimark D, Wanner C, Tangri N: Predicting mortality in incident dialysis patients: an analysis of the United Kingdom Renal Registry. Am J Kidney Dis 2011;57:894-902.

-97 Ivory SE, Polkinghorne KR, Khandakar Y, Kasza J, Zoungas S, Steenkamp R, Roderick P, Wolfe R: Predicting 6-month mortality risk of patients commencing dialysis treatment for end-stage kidney disease. Nephrol Dial Transplant 2017 DOI: 10.1093/ndt/gfw383.

-98 Floege J, Gillespie IA, Kronenberg F, Anker SD, Gioni I, Richards S, Pisoni RL, Robinson BM, Marcelli D, Froissart M, Eckardt K-U: Development and validation of a predictive mortality risk score from a European hemodialysis cohort. Kidney Int 2015;87:996-1008.

99 Couchoud CG, Beuscart J-BR, Aldigier J-C, Brunet PJ, Moranne OP, REIN registry: Development of a risk stratification algorithm to improve patient-centered care and decision making for incident elderly patients with end-stage renal disease. Kidney Int 2015;88:1178-1186.

100 Dusseux E, Albano L, Fafin C, Hourmant M, Guérin O, Couchoud C, Moranne O: A simple clinical tool to inform the decision-making process to refer elderly incident dialysis patients for kidney transplant evaluation. Kidney Int 2015;88:121-129.

101 Reuter S, Reiermann S, Malyar V, Schütte-Nütgen K, Schmidt R, Pavenstädt H, Reinecke H, Suwelack B: A Comparison of Different Algorithms for the Assessment of Cardiovascular Risk in Patients at Waiting List for Kidney Transplantation. PLoS One 2016;11:e0161927.

102 Ma TK-W, Chow KM, Kwan BC-H, Ng JK-C, Pang W-F, Leung CB, Li PK-T, Szeto CC: The choice of comorbidity scoring system in Chinese peritoneal dialysis patients. Clin Exp Nephrol 2017. DOI: 10.1007/s10157-0171418-5.

103 Yang H, Chen Y-H, Hsieh TF, Chuang SY, Wu MJ: Prediction of Mortality in Incident Hemodialysis Patients: A Validation and Comparison of CHADS2, CHA2DS2, and CCI Scores. PLoS One 2016;11:e0154627.

104 Kasiske BL, Chakkera HA, Roel J: Explained and unexplained ischemic heart disease risk after renal transplantation. J Am Soc Nephrol 2000;11:1735-1743.

105 Silver SA, Huang M, Nash MM, Prasad GVR: Framingham risk score and novel cardiovascular risk factors underpredict major adverse cardiac events in kidney transplant recipients. Transplantation 2011;92:183189. 


\section{Kidney \\ Blood Pressure Research}

106 McAdams-DeMarco MA, King EA, Luo X, Haugen C, DiBrito S, Shaffer A, Kucirka LM, Desai NM, Dagher NN, Lonze BE, Montgomery RA, Walston J, Segev DL: Frailty, Length of Stay, and Mortality in Kidney Transplant Recipients: A National Registry and Prospective Cohort Study. Ann Surg 2016. DOI: 10.1097/ SLA.0000000000002025.

107 Ojo AO, Hanson JA, Wolfe RA, Leichtman AB, Agodoa LY, Port FK: Long-term survival in renal transplant recipients with graft function. Kidney Int 2000;57:307-313.

108 Hernández D, Rufino M, Bartolomei S, Lorenzo V, González-Rinne A, Torres A: A novel prognostic index for mortality in renal transplant recipients after hospitalization. Transplantation 2005;79:337-343.

109 Jassal SV, Schaubel DE, Fenton SSA: Baseline comorbidity in kidney transplant recipients: a comparison of comorbidity indices. Am J Kidney Dis 2005;46:136-142.

110 Jassal SV, Schaubel DE, Fenton SSA: Predicting mortality after kidney transplantation: a clinical tool. Transpl Int 2005;18:1248-1257.

111 Wu C, Evans I, Joseph R, Shapiro R, Tan H, Basu A, Smetanka C, Khan A, McCauley J, Unruh M: Comorbid conditions in kidney transplantation: association with graft and patient survival. J Am Soc Nephrol 2005;16:3437-3444.

112 Cardinal H, Hébert M-J, Rahme E, Houde I, Baran D, Masse M, Boucher A, Le Lorier J: Modifiable factors predicting patient survival in elderly kidney transplant recipients. Kidney Int 2005;68:345-351.

113 Schaeffner ES, Födinger M, Kramar R, Frei U, Hörl WH, Sunder-Plassmann G, Winkelmayer WC: Prognostic associations between lipid markers and outcomes in kidney transplant recipients. Am J Kidney Dis 2006;47:509-517.

114 Lin RS, Horn SD, Hurdle JF, Goldfarb-Rumyantzev AS: Single and multiple time-point prediction models in kidney transplant outcomes. J Biomed Inform 2008;41:944-952.

115 Cosio FG, Hickson LJ, Griffin MD, Stegall MD, Kudva Y: Patient survival and cardiovascular risk after kidney transplantation: the challenge of diabetes. Am J Transplant 2008;8:593-599.

-116 Moers C, Kornmann NSS, Leuvenink HGD, Ploeg RJ: The influence of deceased donor age and old-for-old allocation on kidney transplant outcome. Transplantation 2009;88:542-552.

117 Hernández D, Sánchez-Fructuoso A, González-Posada JM, Arias M, Campistol JM, Rufino M, Morales JM, Moreso F, Pérez G, Torres A, Serón D, Spanish Late Allograft Dysfunction Study Group: A Novel Risk Score for Mortality in Renal Transplant Recipients Beyond the First Posttransplant Year. Transplantation 2009;88:803-809.

118 Heldal K, Hartmann A, Leivestad T, Svendsen M V, Foss A, Lien B, Midtvedt K: Clinical outcomes in elderly kidney transplant recipients are related to acute rejection episodes rather than pretransplant comorbidity. Transplantation 2009;87:1045-1051.

119 Machnicki G, Pinsky B, Takemoto S, Balshaw R, Salvalaggio PR, Buchanan PM, Irish W, Bunnapradist S, Lentine KL, Burroughs TE, Brennan DC, Schnitzler MA: Predictive ability of pretransplant comorbidities to predict long-term graft loss and death. Am J Transplant 2009;9:494-505.

120 Israni AK, Snyder JJ, Skeans MA, Peng Y, Maclean JR, Weinhandl ED, Kasiske BL, PORT Investigators: Predicting coronary heart disease after kidney transplantation: Patient Outcomes in Renal Transplantation (PORT) Study. Am J Transplant 2010;10:338-353.

121 Kasiske BL, Israni AK, Snyder JJ, Skeans MA, Peng Y, Weinhandl ED: A simple tool to predict outcomes after kidney transplant. Am J Kidney Dis 2010;56:947-960.

122 Soveri I, Holme I, Holdaas H, Budde K, Jardine AG, Fellström B: A cardiovascular risk calculator for renal transplant recipients. Transplantation 2012;94:57-62.

-123 Dahle DO, Eide IA, Åsberg A, Leivestad T, Holdaas H, Jenssen TG, Fagerland MW, Pihlstrøm H, Mjøen G, Hartmann A: Aortic Stiffness in a Mortality Risk Calculator for Kidney Transplant Recipients. Transplantation 2015;99:1730-1737.

124 Laging M, Kal-van Gestel JA, van de Wetering J, IJzermans JNM, Betjes MGH, Weimar W, Roodnat JI: A High Comorbidity Score Should Not be a Contraindication for Kidney Transplantation. Transplantation 2016;100:400-406.

125 Lorent M, Giral M, Pascual M, Koller MT, Steiger J, Trébern-Launay K, Legendre C, Kreis H, Mourad G, Garrigue V, Rostaing L, Kamar N, Kessler M, Ladrière M, Morelon E, Buron F, Golshayan D, Foucher Y: Mortality Prediction after the First Year of Kidney Transplantation: An Observational Study on Two European Cohorts. PLoS One 2016;11:e0155278. 


\section{Kidney Blood Pressure Research}

Hernández et al.: Mortality in Elderly Waiting-List and Kidney Transplant Patients

126 Zafar MN, Wong G, Aziz T, Abbas K, Rizvi Sa: A living donor risk model for predicting kidney allograft and patient survival in an emerging economy. Nephrology (Carlton) 2016. DOI: 10.1111/nep.12983.

-127 Molnar MZ, Nguyen DV, Chen Y, Ravel V, Streja E, Krishnan M, Kovesdy CP, Mehrotra R, Kalantar-Zadeh K: Predictive Score for Posttransplantation Outcomes. Transplantation 2017;101:1353-1364.

-128 Seoane-Pillado MT, Pita-Fernández S, Valdés-Cañedo F, Seijo-Bestilleiro R, Pértega-Díaz S, Fernández-Rivera C, Alonso-Hernández Á, González-Martín C, Balboa-Barreiro V: Incidence of cardiovascular events and associated risk factors in kidney transplant patients: a competing risks survival analysis. BMC Cardiovasc Disord 2017;17:72.

129 Bergler T, Hutchinson JA: Tools for Predicting Kidney Transplant Outcomes. Transplantation 2017;101:1958-1959.

130 Snyder JJ, Salkowski N, Kim SJ, Zaun D, Xiong H, Israni AK, Kasiske BL: Developing Statistical Models to Assess Transplant Outcomes Using National Registries. Transplantation 2016;100:288-294. 